\title{
Deciphering the Lipid Architecture of the Rat
}

\section{Sciatic Nerve Using Imaging Mass Spectrometry}

Roberto Fernández, ${ }^{1}$ Victor Carriel,,${ }^{2}$ Sergio Lage, ${ }^{1}$ Jone Garate, ${ }^{1}$ Javier Diez-Garcia,${ }^{3}$ Begoña Ochoa, ${ }^{4}$ Begoña Castro, ${ }^{3}$ Miguel Alaminos, ${ }^{2}$ and José A. Fernández ${ }^{1 *}$

${ }^{1}$ Department of Physical Chemistry, Faculty of Science and Technology, ${ }^{4}$ Department of Physiology, Faculty of Medicine and Dentistry, University of the Basque Country UPV/EHU, 48940 Leioa, Spain.

${ }^{2}$ Tissue Engineering Group, Department of Histology, Faculty of Medicine, University of Granada, 18012 Granada, Spain.

${ }^{3}$ Histocell, S.L., Bizkaia Technology Park 800, 48160 Derio, Spain.

KEYWORDS: Imaging mass spectrometry, lipidomics, peripheral nervous system.

Corresponding author:

*José A. Fernández

CD1.S1.10

Departamento de Química Física

Facultad de Ciencia y Tecnología

Universidad del País Vasco (UPV/EHU)

Barrio Sarriena s/n, 48940 Leioa

Spain

email: josea.fernandez@ehu.es

Phone: +34946015387

Fax: +34946013500 
Figure S1. Lysophosphatidylcholine (LPC), phosphatidylcholine (PC) and PC-ether species detected in positive-ion mode in the rat sciatic nerve using MALDI-IMS and their relative abundance in the three areas of the nerve. Those species with an asterisk were confirmed by LC-MS/MS.

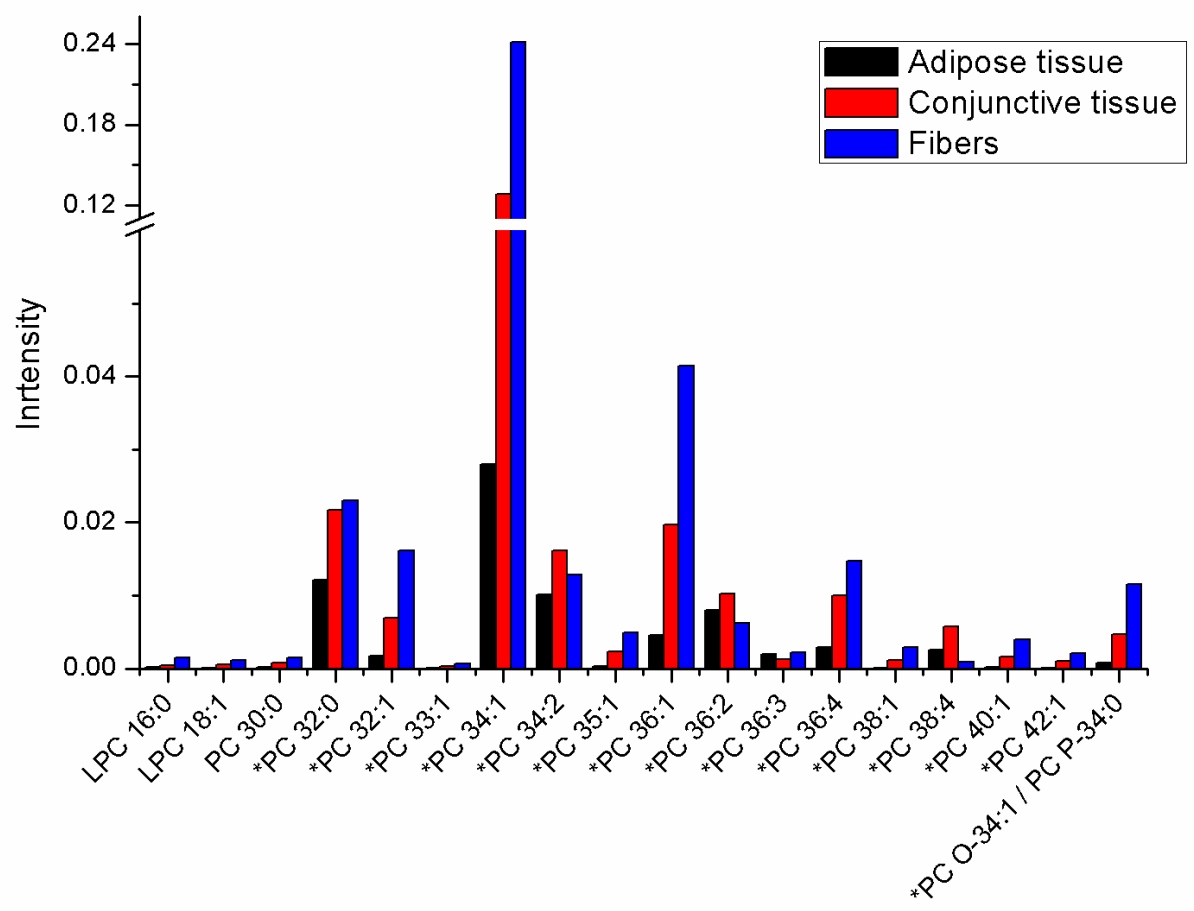

Figure S2. Sphingolipid species detected in positive-ion mode in the rat sciatic nerve using MALDI-IMS and their relative abundance in the three areas of the nerve. Those species with an asterisk were confirmed by LC-MS/MS.

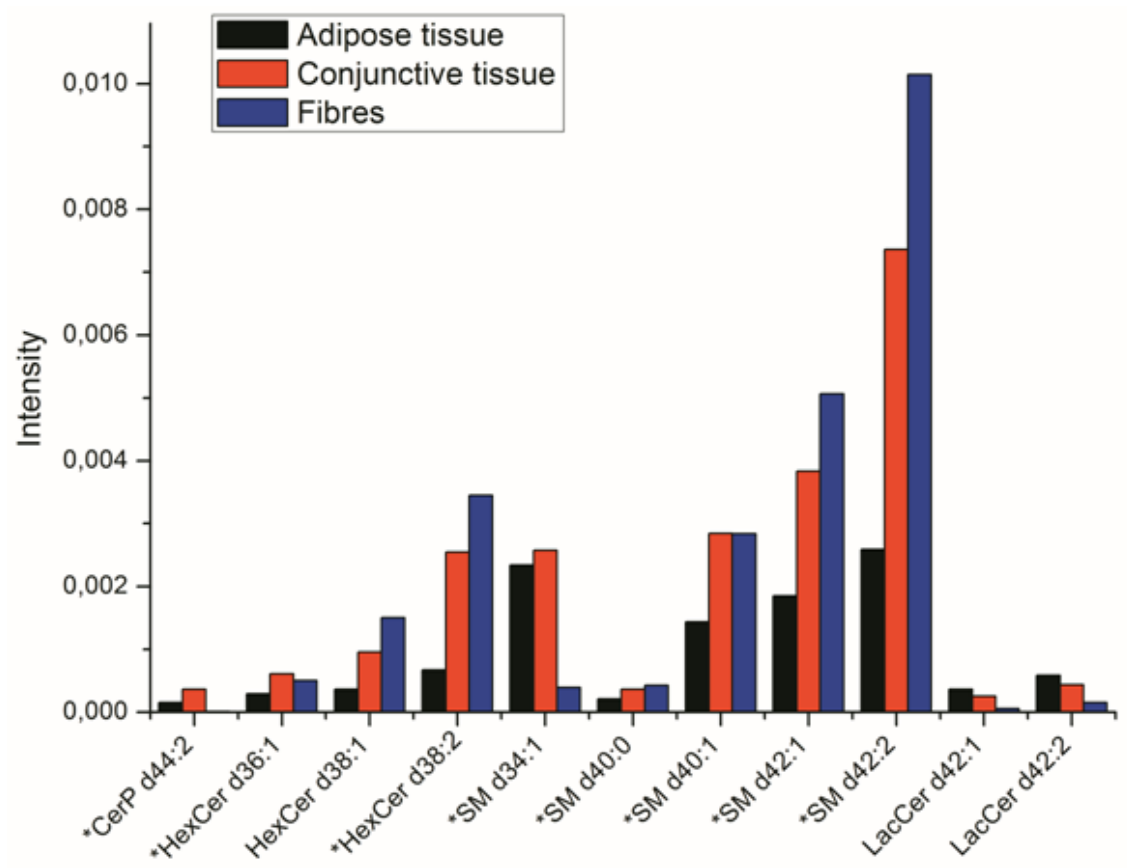


Figure S3. Phosphatidic acid (PA) species detected in positive-ion mode in the rat sciatic nerve using MALDI-IMS and their relative abundance in the three areas of the nerve. Those species with an asterisk were confirmed by LC-MS/MS.

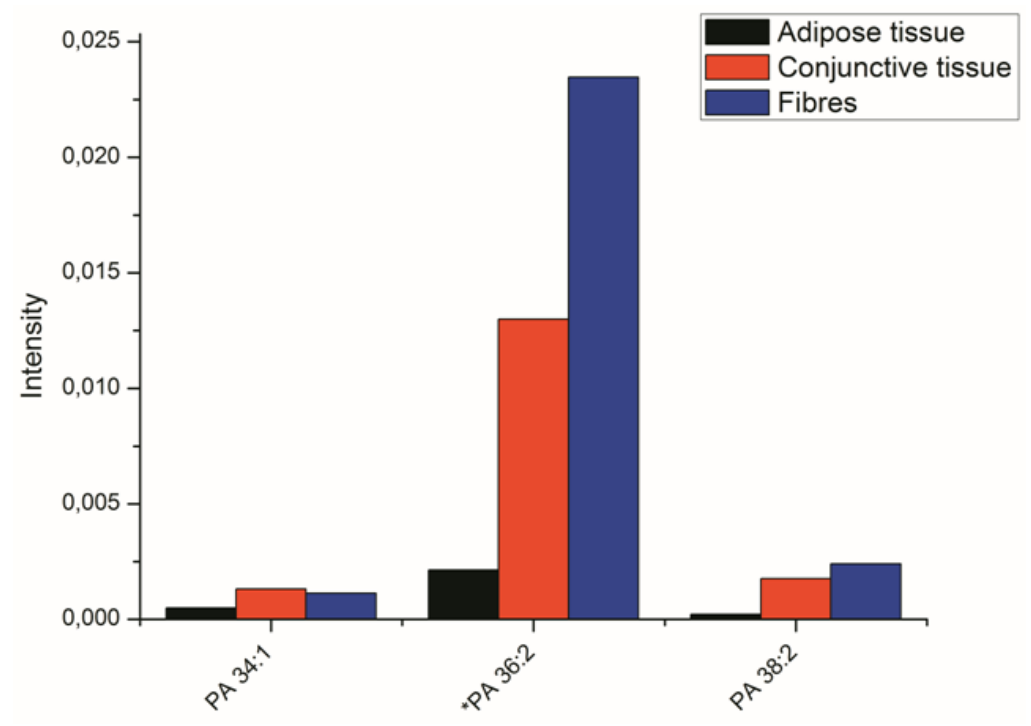

Figure S4. Triglyceride (TG) species detected in positive-ion mode in the rat sciatic nerve using MALDI-IMS and their relative abundance in the three areas of the nerve. Those species with an asterisk were confirmed by LC-MS/MS.

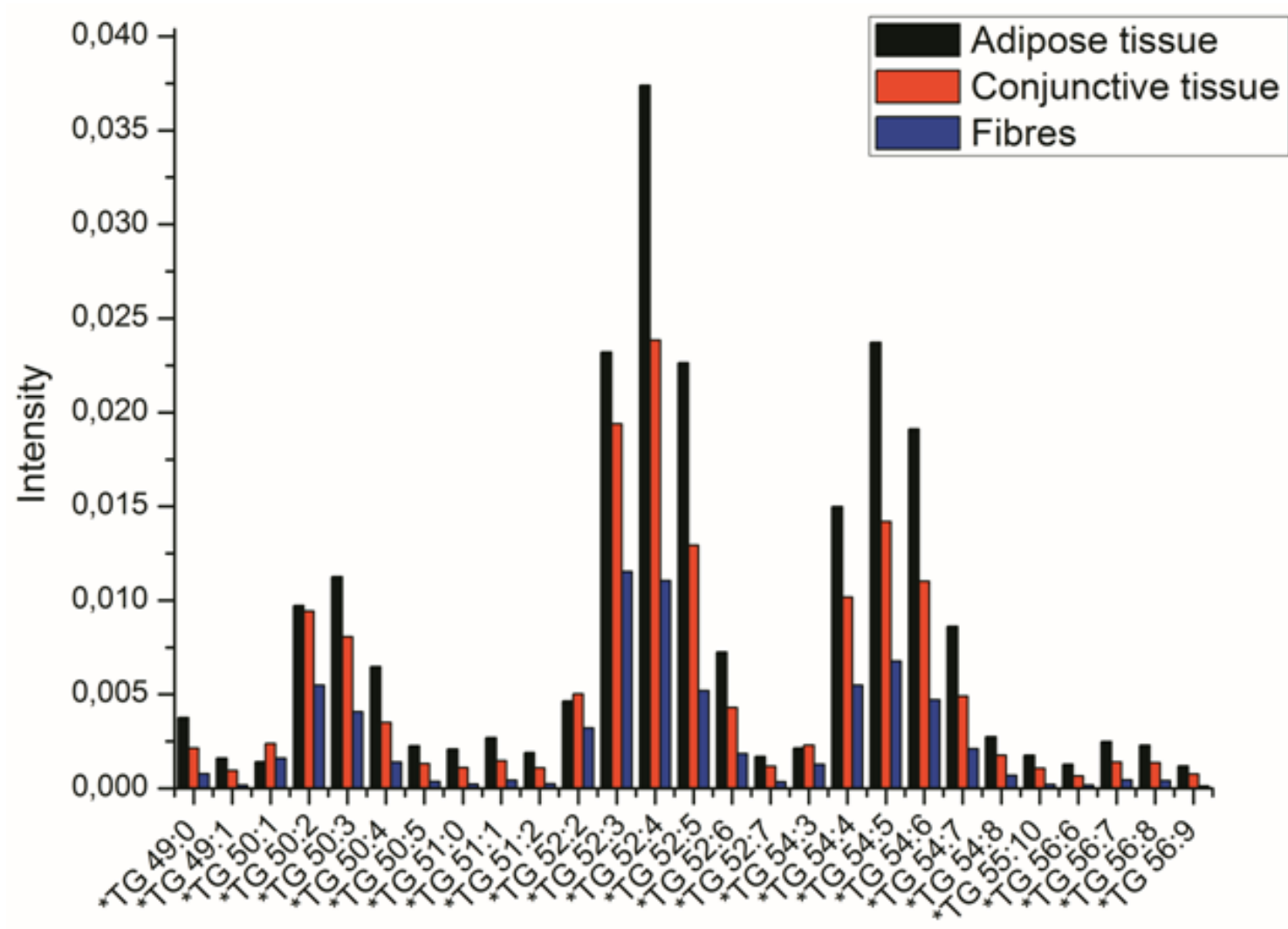


Figure S5. Diglyceride (DG) and DG-ether species detected in positive-ion mode in the rat sciatic nerve using MALDI-IMS and their relative abundance in the three areas of the nerve.

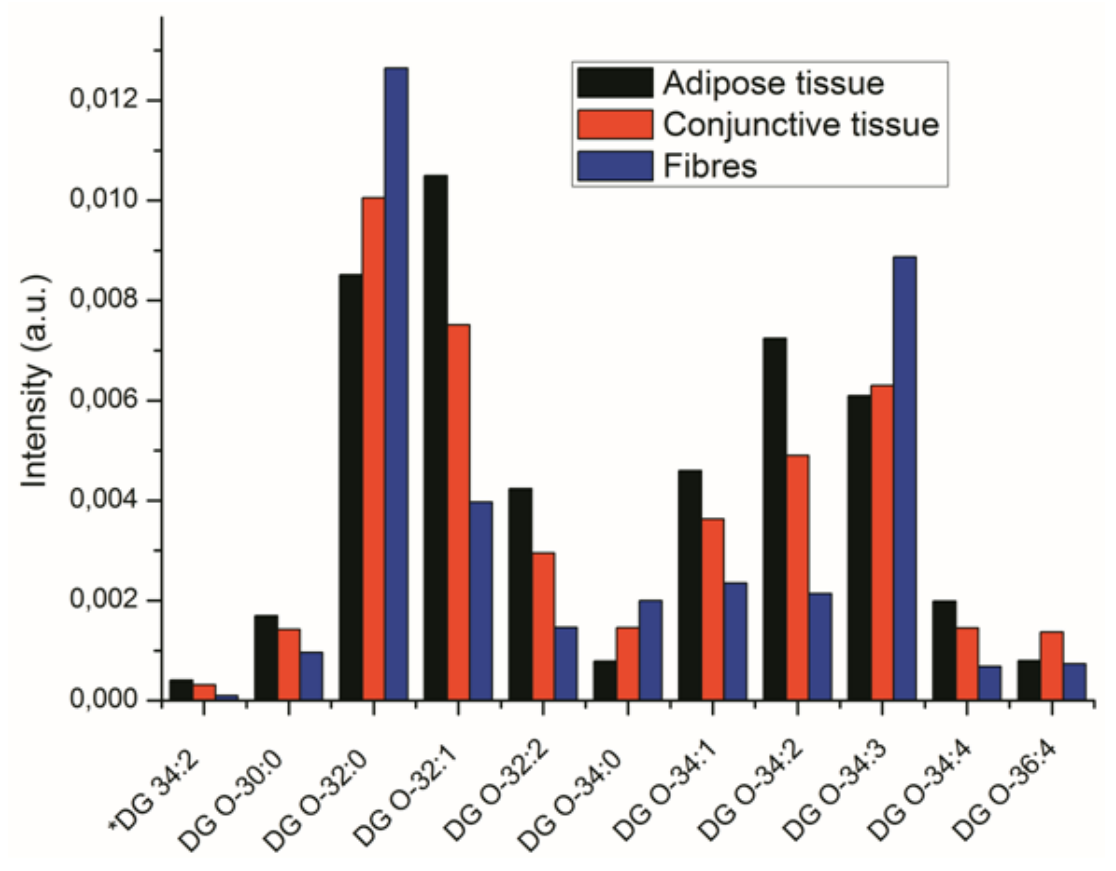

Figure S6. Sulfatide (SFT) species detected in negative-ion mode in the rat sciatic nerve using MALDI-IMS and their relative abundance in the three areas of the nerve. Those species with an asterisk were confirmed by LC-MS/MS.

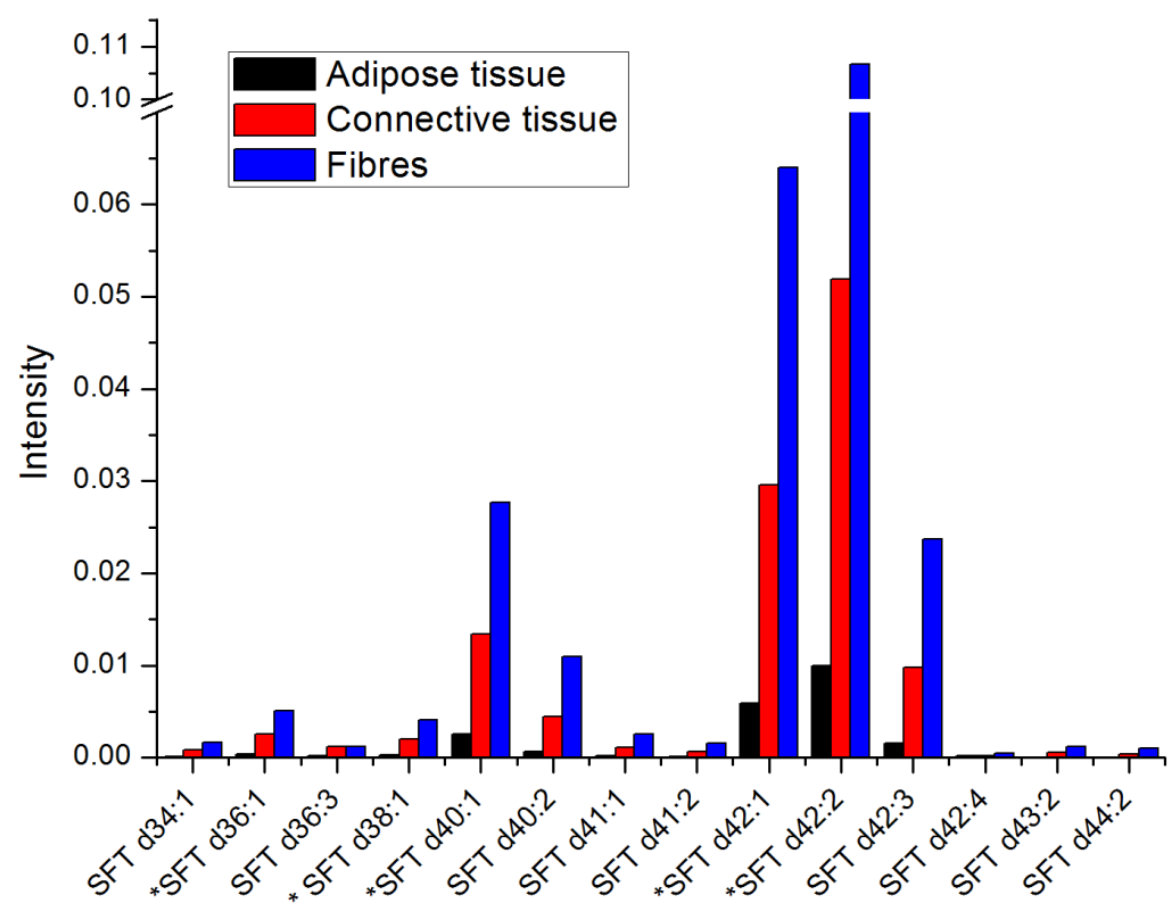


Figure S7. Cerebrosides (gluco- or galacto-cerebrosides, HexCer) species detected in negative-ion mode in the rat sciatic nerve using MALDI-IMS and their relative abundance in the three areas of the nerve. Those species with an asterisk were confirmed by LCMS/MS.

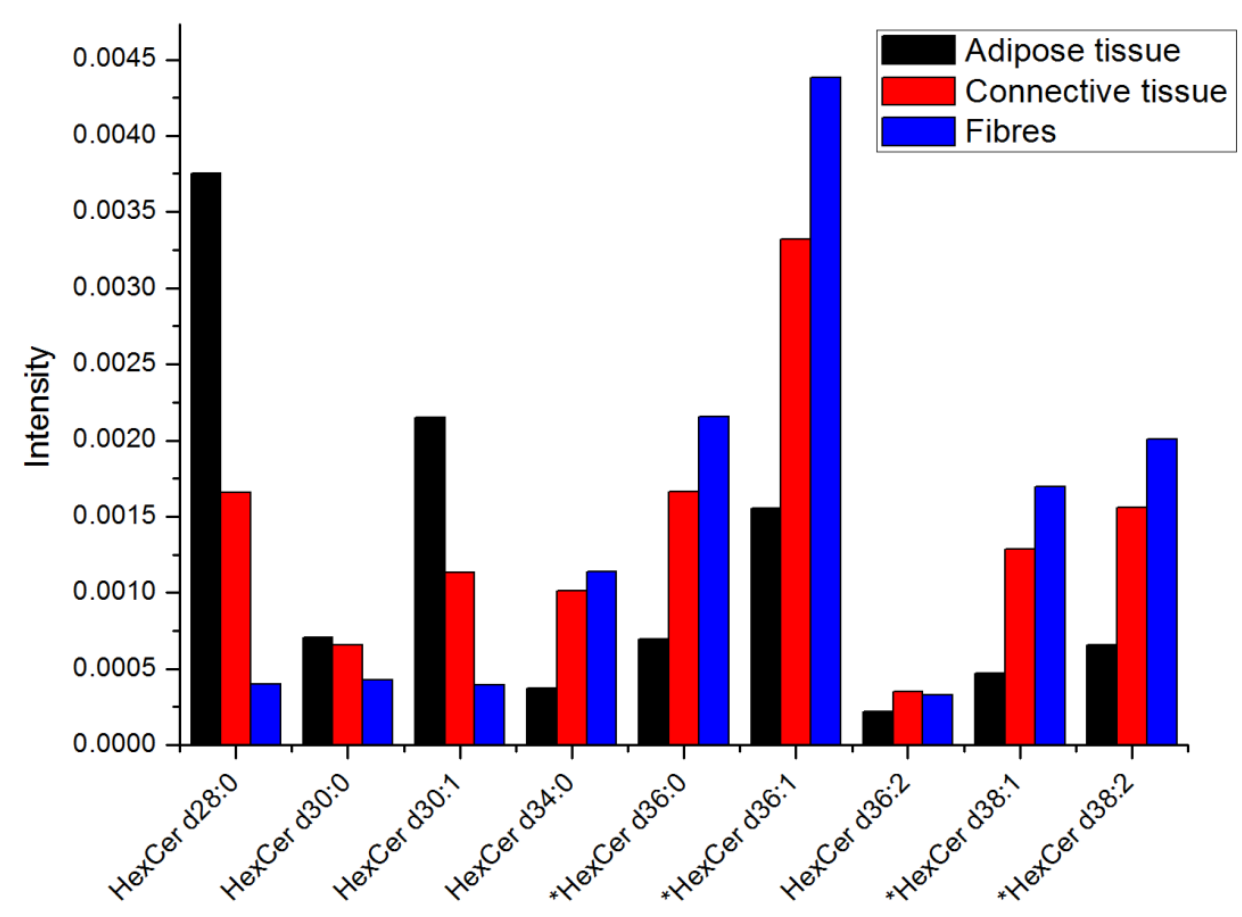

Figure S8. Lysophosphatidylinositol (LPI), phosphatidylinositol (PI) and PI-ether species detected in negative-ion mode in the rat sciatic nerve using MALDI-IMS and their relative abundance in the three areas of the nerve. Those species with an asterisk were confirmed by LC-MS/MS. 


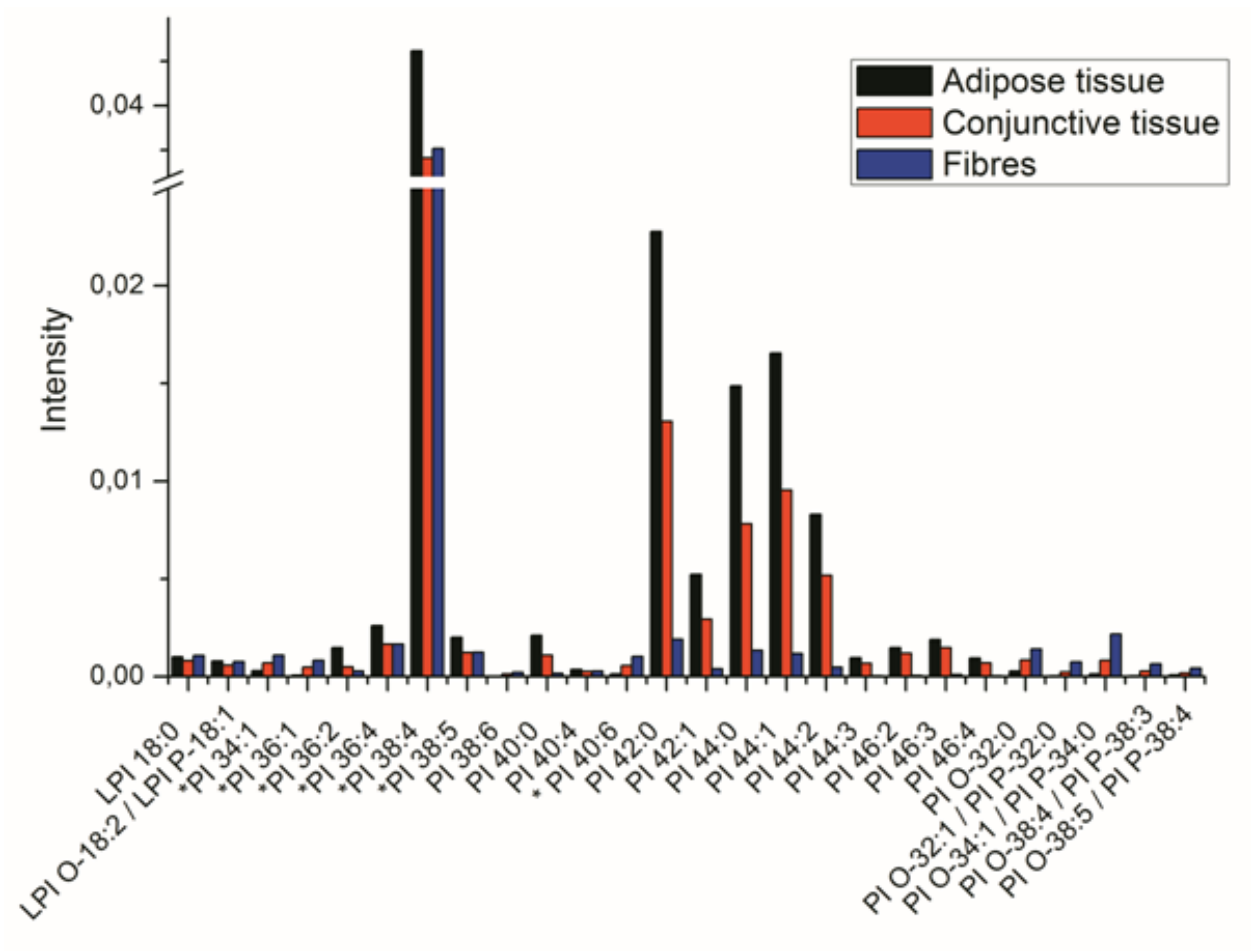

Figure S9. PE-Cer species detected in negative-ion mode in the rat sciatic nerve using MALDI-IMS and their relative abundance in the three areas of the nerve. Those species with an asterisk were confirmed by LC-MS/MS.

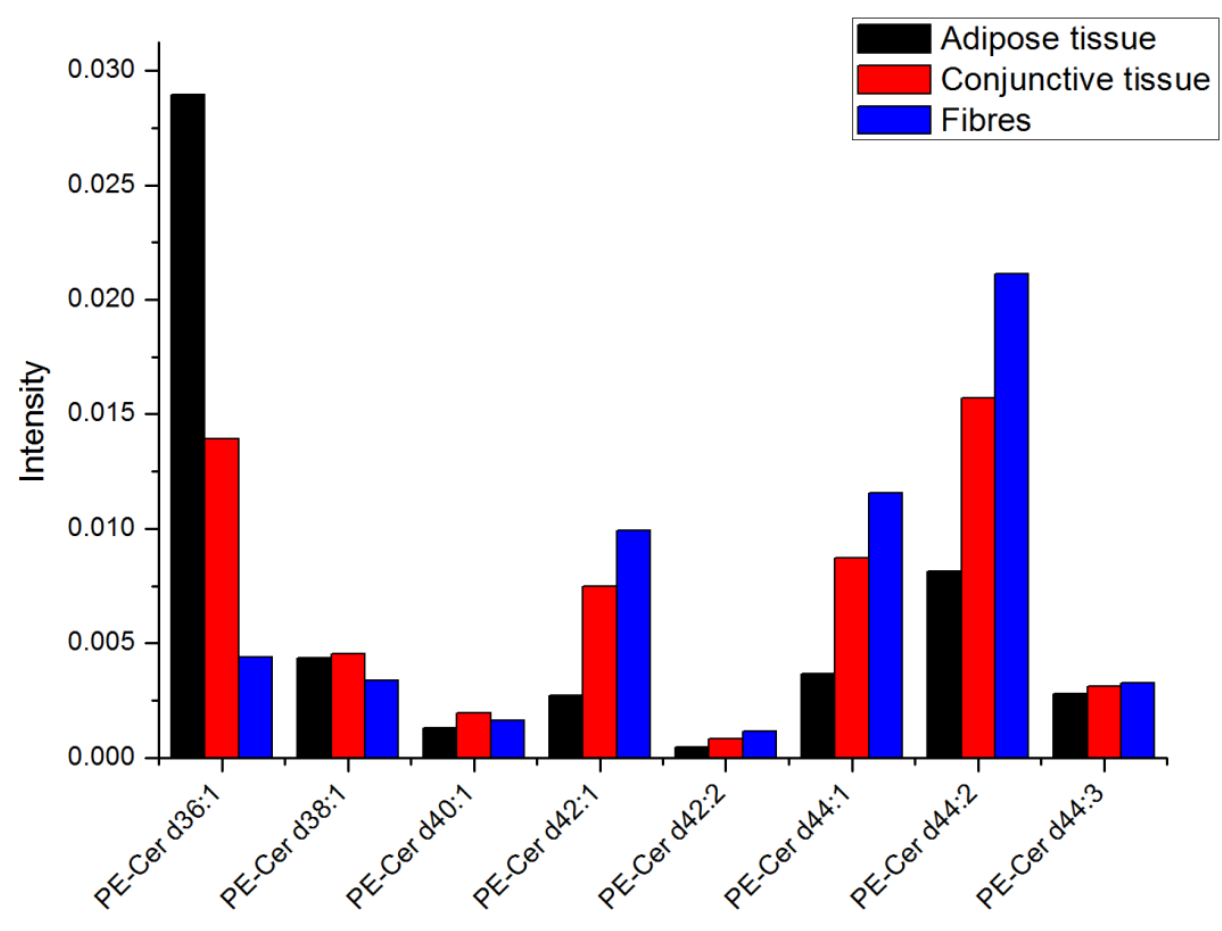


Figure S10. Phosphatidylethanolamine (PE) and PE-ether species detected in negative-ion mode in rat's sciatic nerve using MALDI-IMS and their relative abundance in the three areas of the nerve. Those species with an asterisk were confirmed by LC-MS/MS.

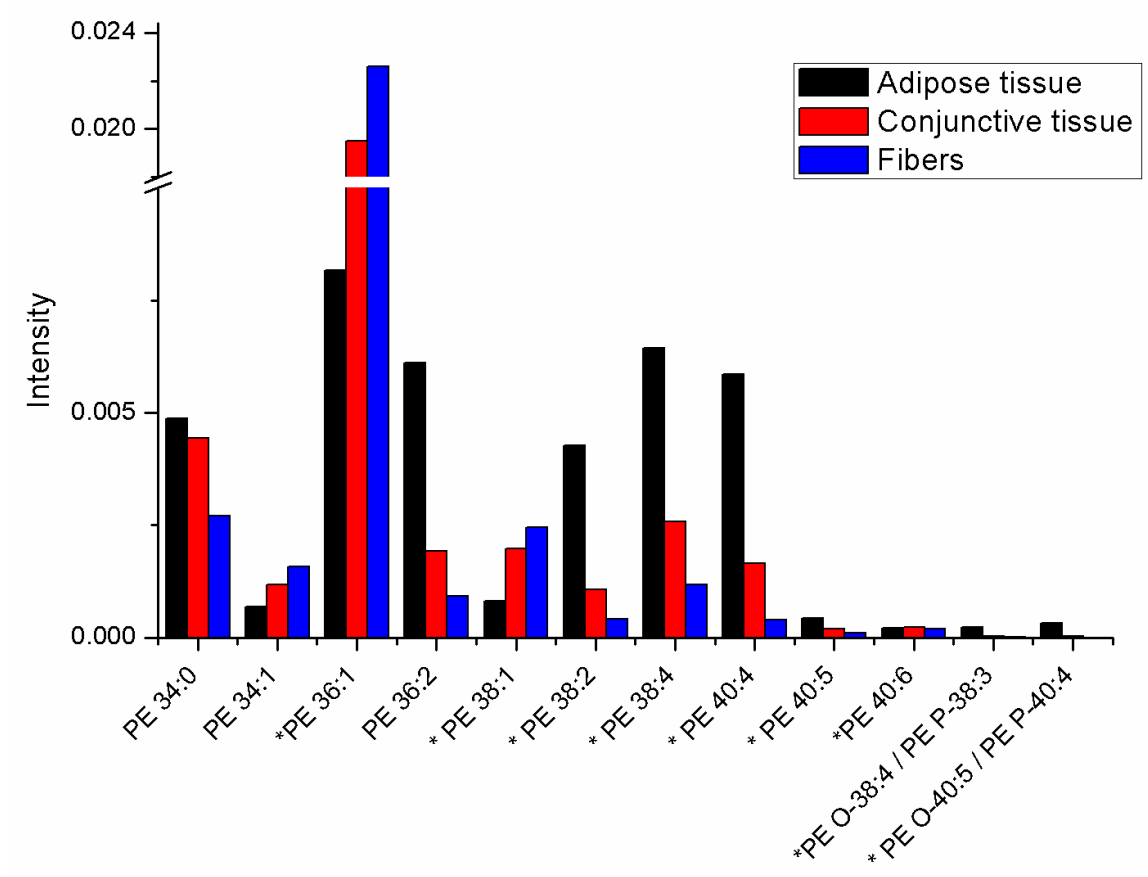

Figure S11. Phosphatidylserine (PS) and PS-ether species detected in negative-ion mode in rat's sciatic nerve using MALDI-IMS and their relative abundance in the three areas of the nerve. Those species with an asterisk were confirmed by LC-MS/MS.

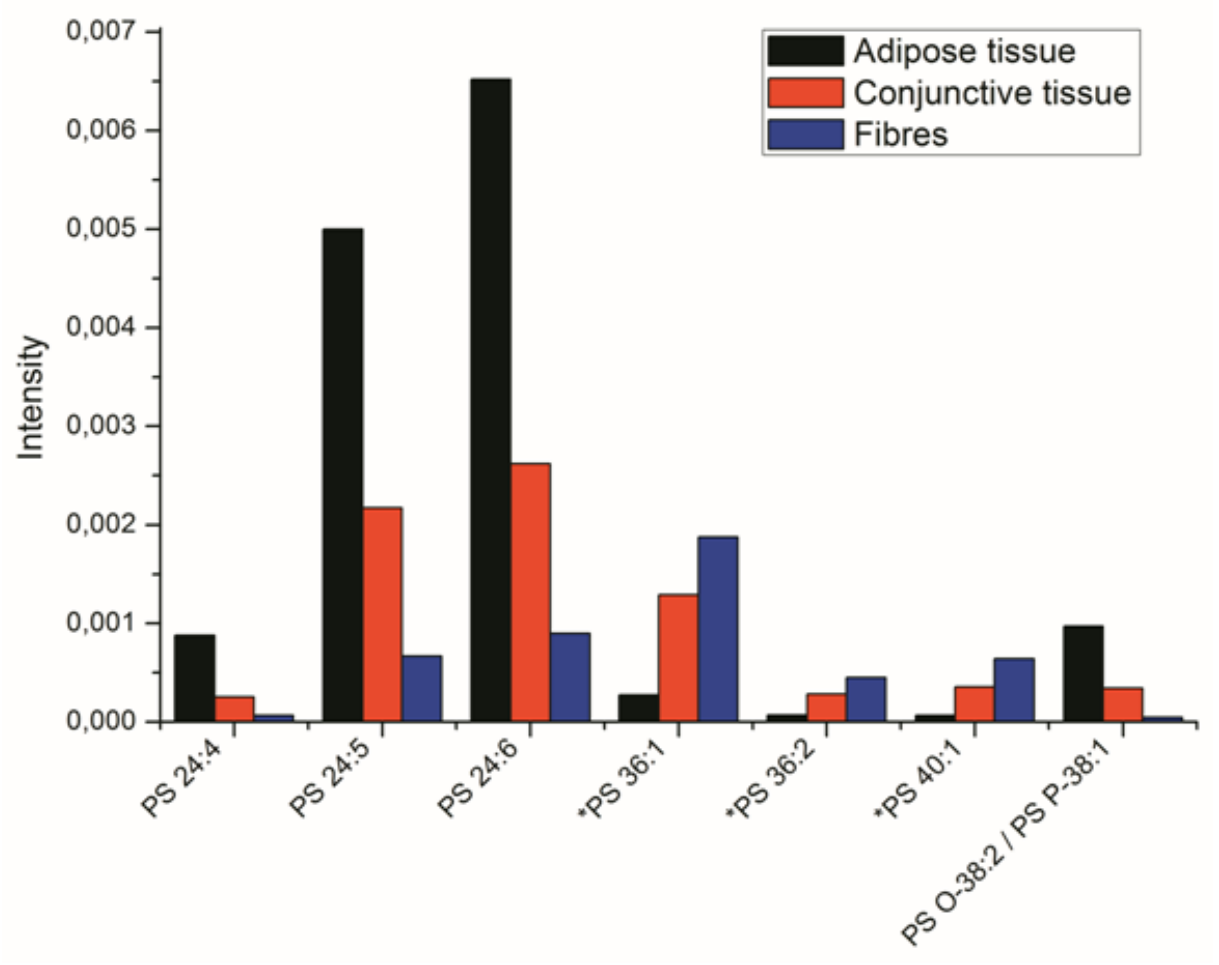


Figure S12. PA species detected in negative-ion mode in rat's sciatic nerve using MALDIIMS and their relative abundance in the three areas of the nerve. Those species with an asterisk were confirmed by LC-MS/MS.

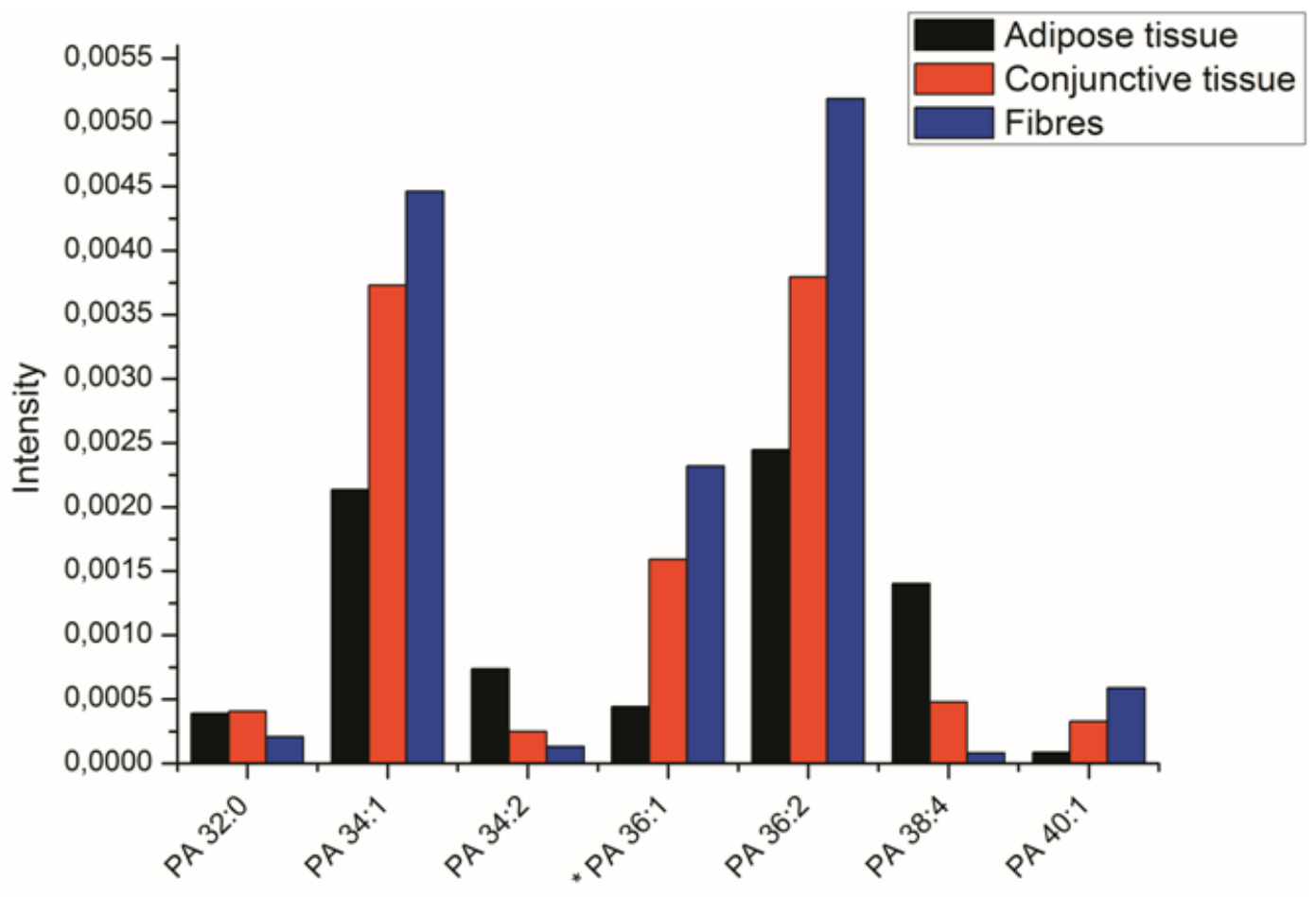

Figure S13. Cer-P species detected negative-ion mode in rat's sciatic nerve using MALDIIMS and their relative abundance in the three areas of the nerve. Those species with an asterisk were confirmed by LC-MS/MS.

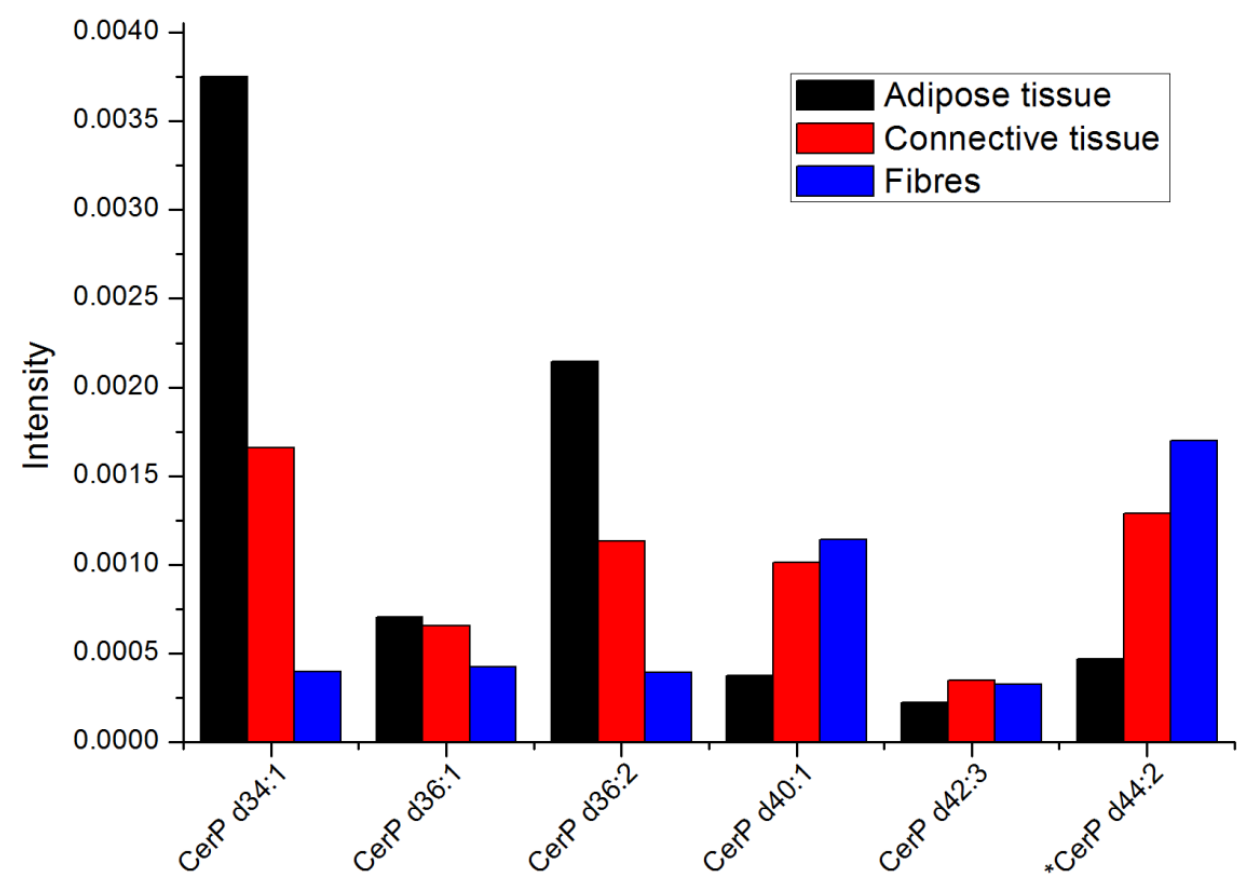


Figure S14. HPLC-MS/MS spectrum of SM (18:1/24:0) at m/z 815.703 and retention time of 6.77 .
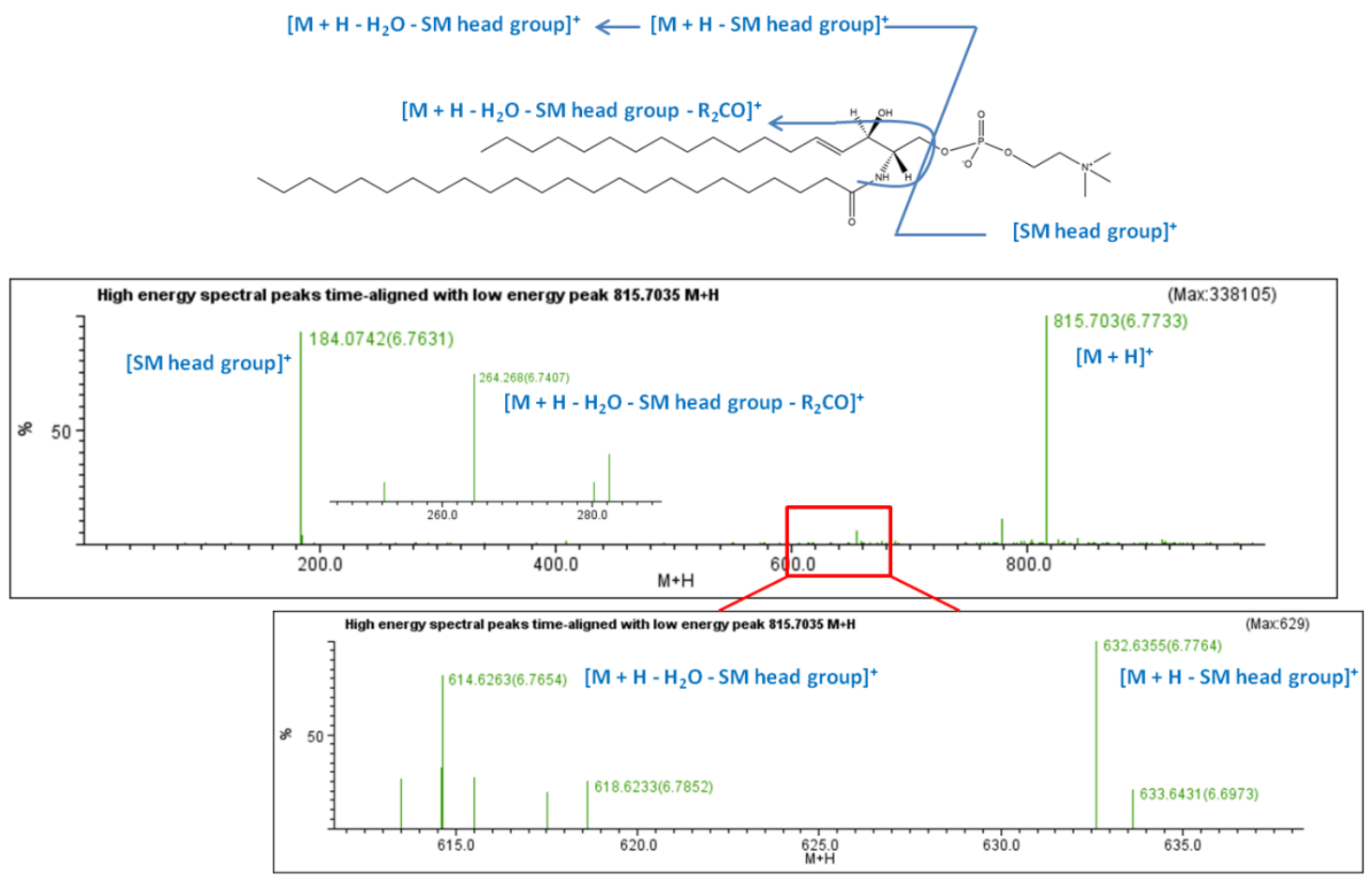

Figure S15. HPLC-MS/MS spectrum of PC (18:1/17:0) at m/z 774.601 and retention time of 5.53 .
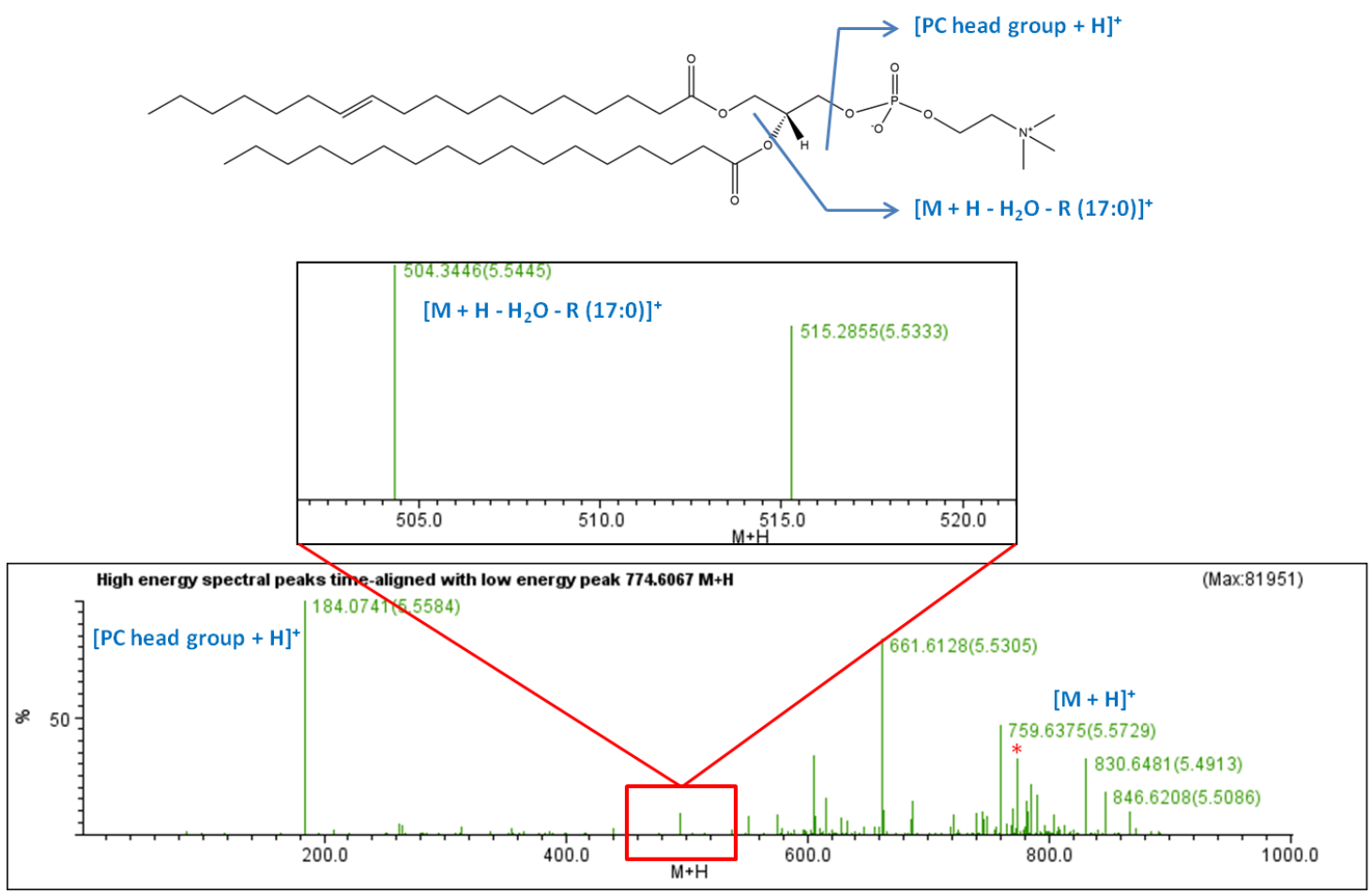
Figure S16. HPLC-MS/MS spectra of PS (18:1/22:0) in both ESI+ (m/z: 846.6208) and ESI- (m/z: 844.6075) at retention time of 5.53.
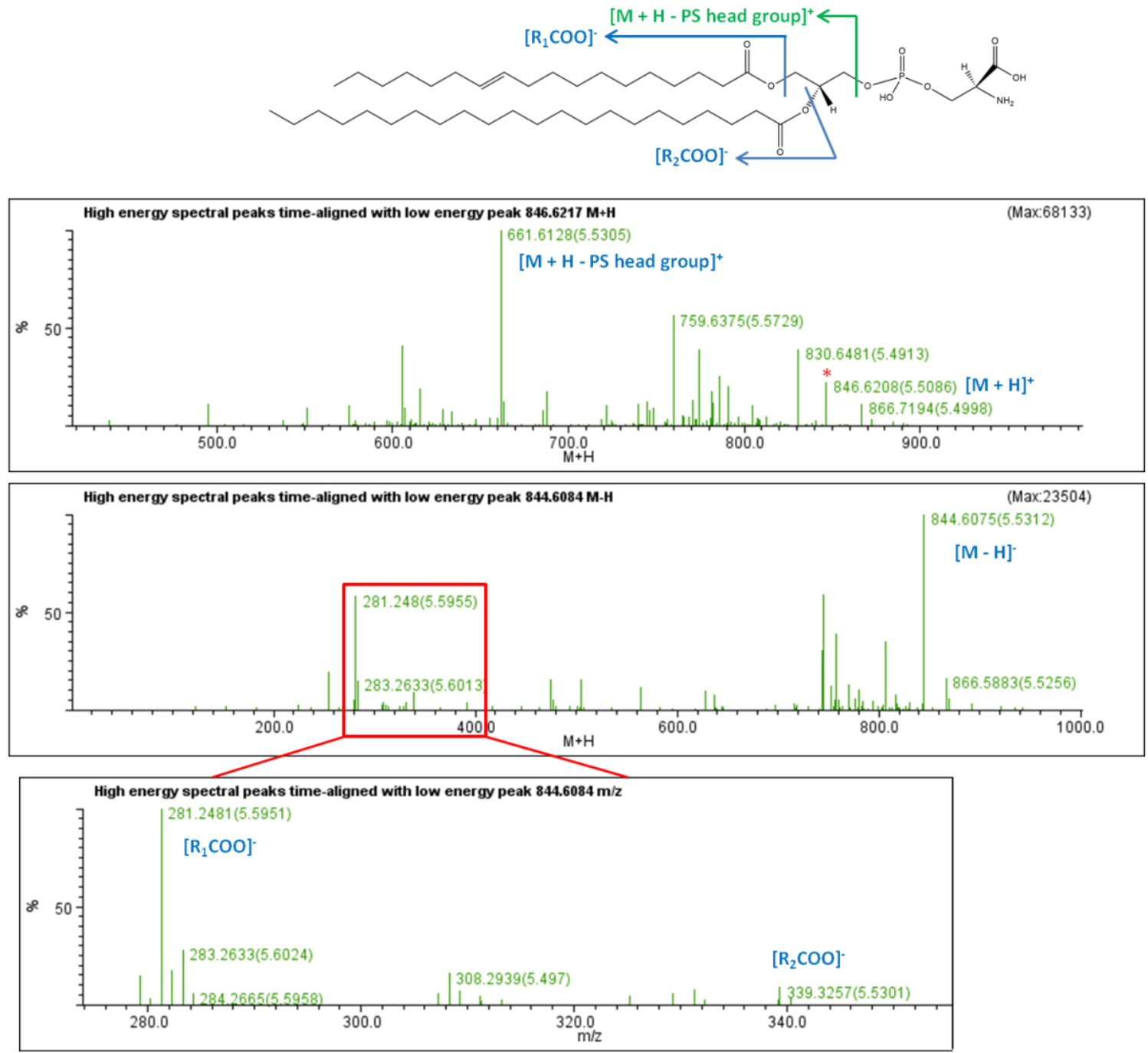
Table S1. Glycerophospholipid molecular species identified in the rat sciatic nerve by IMS or LC-MS/MS.

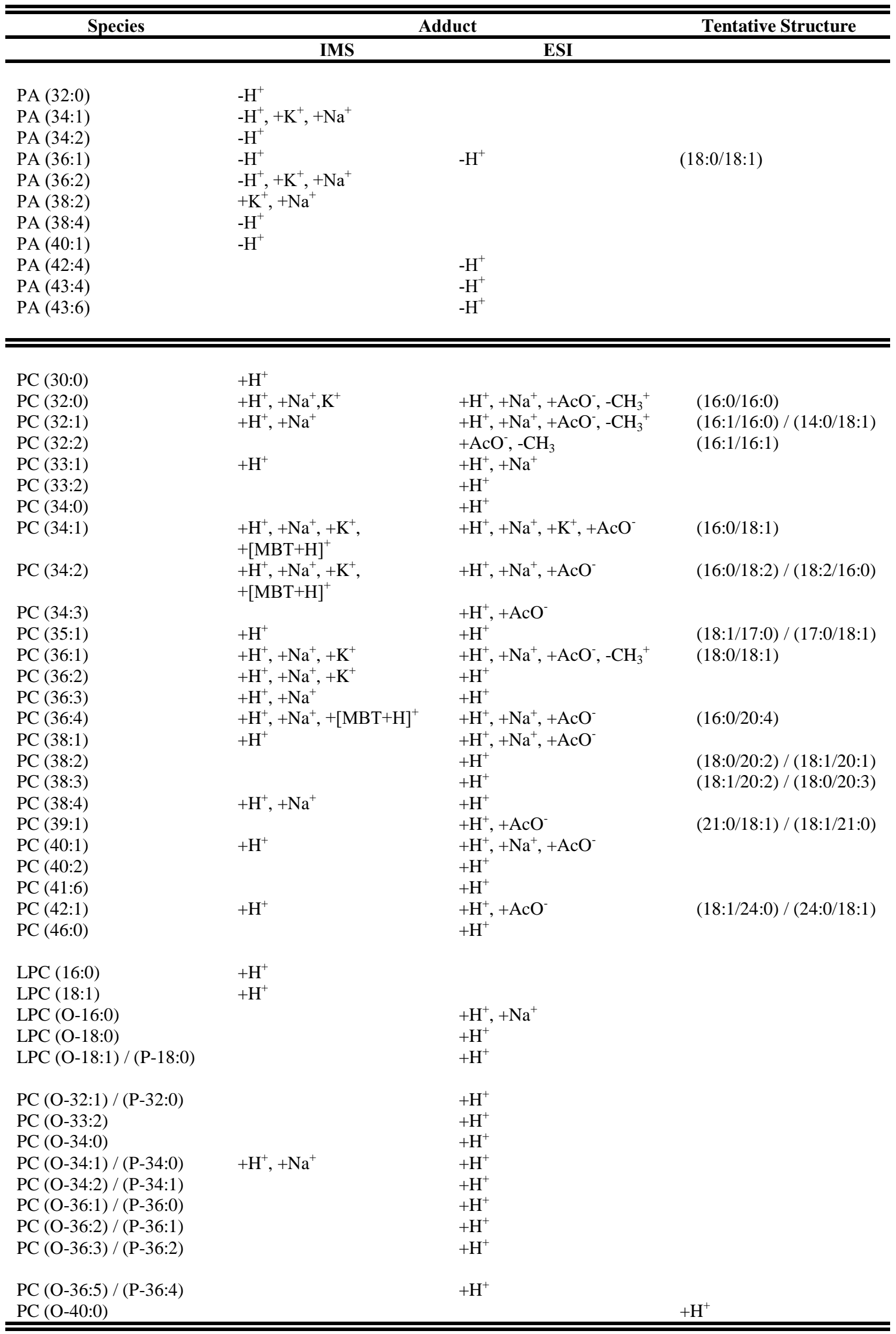


LPE (18:0)

LPE (18:1)

LPE (20:0)

LPE (20:1)

PE (34:0)

PE (34:1)

PE (34:2)

PE (36:1)

PE (36:2)

PE (36:3)

PE (36:4)

PE (38:1)

PE (38:2)

PE (38:4)

PE (38:5)

PE (38:6)

PE (40:1)

PE (40:4)

PE (40:5)

PE (40:6)

PE (42:1)

LPE (O-16:1) / (P-16:0)

LPE (O-18:1) / (P-18:0)

PE (O-32:2) / (P-32:1)

PE (O-32:1) / (P-32:0)

PE (O-33:2) / (P-33:1)

PE (O-34:1) / (P-34:0)

PE (O-34:2) / (P-34:1)

PE (O-34:3) / (P-34:2)

PE (O-34:4) / (P-34:3)

PE (O-35:2) / (P-35:1)

PE $(\mathrm{O}-35: 3) /(\mathrm{P}-35: 2)$

PE (O-36:2) / (P-36:1)

PE (O-36:3) / (P-36:2)

$\mathrm{PE}(\mathrm{O}-36: 4) /(\mathrm{P}-36: 3)$

PE (O-36:5) / (P-36:4)

PE (O-38:0)

PE (O-38:2) / (P-38:1)

$\mathrm{PE}(\mathrm{O}-38: 3) /(\mathrm{P}-38: 2)$

PE (O-38:4) / (P-38:3)

PE (O-38:5) / (P-38:4)

PE (O-38:6) / (P-38:5)

PE (O-38:7) / (P-38:6)

PE $(\mathrm{O}-40: 7) /(\mathrm{P}-40: 6)$

PE (O-40:5) / (P-40:4)

PE (O-40:6) / (P-40:5)

PE (O-41:0)

PE (O-42:1) / (P-42:0)

PE (O-42:0)
$-\mathrm{H}^{+}$
$-\mathrm{H}^{+}$
$-\mathrm{H}^{+}$
$-\mathrm{H}^{+}$

$-\mathrm{H}^{+}$

$-\mathrm{H}^{+},+\mathrm{H}^{+}$

$-\mathrm{H}^{+},+\mathrm{H}^{+}$

$-\mathrm{H}^{+},+\mathrm{H}^{+},+\mathrm{Na}^{+}$

$(16: 1 / 18: 1)$

$(18: 0 / 18: 1)$

$-\mathrm{H}^{+}$

$-\mathrm{H}^{+},+\mathrm{H}^{+}$

$-\mathrm{H}^{+}$

$-\mathrm{H}^{+}$
$-\mathrm{H}^{+}$

$-\mathrm{H}^{+},+\mathrm{H}^{+}$

$-\mathrm{H}^{+},+\mathrm{H}^{+}$

$-\mathrm{H}^{+},+\mathrm{H}^{+}$

$-\mathrm{H}^{+},+\mathrm{H}^{+},+\mathrm{Na}^{+}$

$-\mathrm{H}^{+},+\mathrm{H}^{+}$

$-\mathrm{H}^{+},+\mathrm{H}^{+}$

$-\mathrm{H}^{+}$

$-\mathrm{H}^{+},+\mathrm{H}^{+}$

$-\mathrm{H}^{+}$

$-\mathrm{H}^{+}$

$-\mathrm{H}^{+},+\mathrm{H}^{+}$

$-\mathrm{H}^{+},+\mathrm{H}^{+}$

$-\mathrm{H}^{+} /-\mathrm{H}^{+},+\mathrm{H}^{+}$

$-\mathrm{H}^{+}$

$-\mathrm{H}^{+},+\mathrm{H}^{+}$

$-\mathrm{H}^{+}$

$-\mathrm{H}^{+},+\mathrm{H}^{+}$

$-\mathrm{H}^{+},+\mathrm{H}^{+}$

$-\mathrm{H}^{+},+\mathrm{H}^{+}$

$-\mathrm{H}^{+},+\mathrm{H}^{+}$

$-\mathrm{H}^{+},+\mathrm{H}^{+}$

$-\mathrm{H}^{+},+\mathrm{H}^{+},+\mathrm{Na}^{+}$

$-\mathrm{H}^{+},+\mathrm{H}^{+},+\mathrm{Na}^{+}$

$-\mathrm{H}^{+},+\mathrm{H}^{+},+\mathrm{Na}^{+}$

$-\mathrm{H}^{+}$

$-\mathrm{H}^{+},+\mathrm{H}^{+}$

$-\mathrm{H}^{+},+\mathrm{H}^{+}$

$-\mathrm{H}^{+}$

$-\mathrm{H}^{+},+\mathrm{H}^{+}$

$-\mathrm{H}^{+},+\mathrm{H}^{+}$

$-\mathrm{H}^{+},+\mathrm{H}^{+},+\mathrm{Na}^{+}$

$-\mathrm{H}^{+},+\mathrm{H}^{+}$

$-\mathrm{H}^{+},+\mathrm{H}^{+}$

$-\mathrm{H}^{+}$

$-\mathrm{H}^{+},+\mathrm{H}^{+}$

$-\mathrm{H}^{+},+\mathrm{H}^{+}$

$-\mathrm{H}^{+},+\mathrm{H}^{+}$

$-\mathrm{H}^{+},+\mathrm{Na}^{+}$

$-\mathrm{H}^{+}$
(24:0/18:1)

(18:2/18:1)

(20:0/18:1)/(18:1/20:0)/

(16:0/22:1)

(18:1/20:1)

(18:0/20:4)

$(18: 1 / 20: 4)$

(16:0/22:6)

$(22: 0 / 18: 1) /(20: 1 / 20: 0)$

\begin{tabular}{llll}
\hline \hline & & & \\
PS (24:4) & $-\mathrm{H}^{+}$ & \\
PS (24:5) & $-\mathrm{H}^{+}$ & & \\
PS (24:6) & $-\mathrm{H}^{+}$ & & \\
PS (34:1) & & $-\mathrm{H}^{+},+\mathrm{H}^{+}$ & $(16: 0 / 18: 1)$ \\
PS (36:1) & $-\mathrm{H}^{+}$ & $+\mathrm{H}^{+},+\mathrm{Na}^{+}$ & $(18: 0 / 18: 1)$ \\
PS (36:2) & $-\mathrm{H}^{+}$ & $-\mathrm{H}^{+},+\mathrm{H}^{+},+\mathrm{Na}^{+}$ & $(18: 1 / 18: 1) /(18: 0 / 18: 2)$ \\
PS (38:1) & & $-\mathrm{H}^{+},+\mathrm{H}^{+}$ & $(18: 1 / 20: 0)$ \\
PS (38:2) & & $-\mathrm{H}^{+}$ & $(18: 0 / 20: 2) /(18: 1 / 20: 1)$ \\
PS (38:3) & & $-\mathrm{H}^{+},+\mathrm{H}^{+}$ & $(18: 1 / 20: 3) /(18: 0 / 20: 4)$ \\
PS (38:4) & & $-\mathrm{H}^{+},+\mathrm{H}^{+}$ &
\end{tabular}


PS (38:5)

PS (40:1)

PS (40:2)

PS (40:4)

PS (42:1)

PS (42:4)

PS (42:5)
$-\mathrm{H}^{+}$

$-\mathrm{H}^{+},+\mathrm{H}^{+},+\mathrm{Na}^{+}$

$-\mathrm{H}^{+},+\mathrm{H}^{+}$

$-\mathrm{H}^{+},+\mathrm{H}^{+}$

$-\mathrm{H}^{+},+\mathrm{H}^{+}$

$-\mathrm{H}^{+},+\mathrm{H}^{+}$

$-\mathrm{H}^{+},+\mathrm{H}^{+}$

$(18: 1 / 22: 0)$

$(18: 0 / 22: 4) /(20: 0 / 20: 4)$

$(22: 1 / 20: 4) /(20: 1 / 22: 4)$

PS (O-38:2) / (P-38:1)

$-\mathrm{H}^{+}$

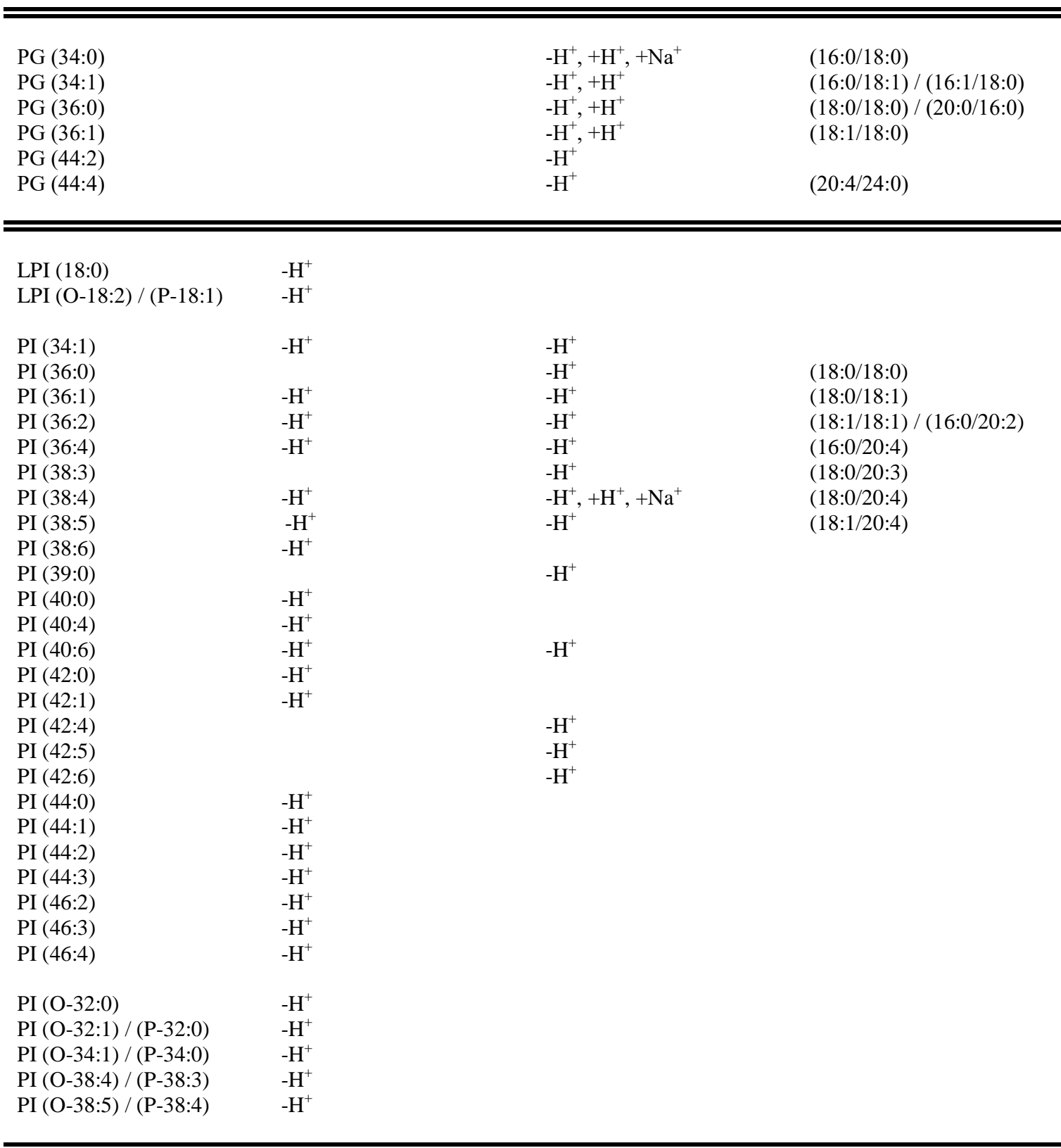


Table S2. Sphingolipid molecular species identified in the rat sciatic nerve by IMS or LCMS/MS.

\begin{tabular}{|c|c|c|c|}
\hline Species & & uct & Tentative Structure \\
\hline & IMS & ESI & \\
\hline Cer (d38:0) & & $+\mathrm{H}^{+}$ & $(18: 0 / 20: 0)$ \\
\hline Cer (d40:0) & & $+\mathrm{H}^{+}$ & $(18: 0 / 22: 0)$ \\
\hline Cer (d40:1) & & $+\mathrm{H}^{+}$ & $(18: 1 / 22: 0)$ \\
\hline Cer (d40:2) & & $+\mathrm{H}^{+}$ & $(18: 1 / 22: 1)$ \\
\hline Cer (d42:0) & & $+\mathrm{H}^{+}$ & $(18: 0 / 24: 0)$ \\
\hline Cer (d42:1) & & $+\mathrm{H}^{+}$ & $(18: 1 / 24: 0)$ \\
\hline Cer (d42:2) & & $+\mathrm{H}^{+}$ & $(18: 1 / 24: 1)$ \\
\hline
\end{tabular}

$\begin{array}{lll}\text { CerP }(\mathrm{d} 34: 1) & -\mathrm{H}^{+} & \\ \operatorname{CerP}(\mathrm{d} 36: 1) & -\mathrm{H}^{+} & \\ \operatorname{CerP}(\mathrm{d} 36: 2) & -\mathrm{H}^{+} & \\ \operatorname{CerP}(\mathrm{d} 40: 1) & -\mathrm{H}^{+} & -\mathrm{H}^{+} \\ \operatorname{CerP}(\mathrm{d} 42: 3) & -\mathrm{H}^{+},+\mathrm{K}^{+} & +\mathrm{H}^{+}\end{array}$

\begin{tabular}{|c|c|c|c|}
\hline $\mathrm{SM}(\mathrm{d} 34: 0)$ & & $+\mathrm{H}^{+}$ & $(18: 0 / 16: 0)$ \\
\hline $\mathrm{SM}(\mathrm{d} 34: 1)$ & $+\mathrm{H}^{+},+\mathrm{Na}^{+},+\mathrm{K}^{+}$ & $+\mathrm{H}^{+},+\mathrm{Na}^{+}$ & $(18: 1 / 16: 0)$ \\
\hline $\mathrm{SM}(\mathrm{d} 36: 1)$ & & $+\mathrm{H}^{+},+\mathrm{Na}^{+}$ & (18:1/18:0) \\
\hline $\mathrm{SM}(\mathrm{d} 36: 2)$ & & $+\mathrm{H}^{+}$ & $(18: 1 / 18: 1) /(18: 2 / 18: 0)$ \\
\hline SM (d38:0) & & $+\mathrm{H}^{+}$ & \\
\hline SM (d38:1) & & $+\mathrm{H}^{+},+\mathrm{Na}^{+},+\mathrm{AcO}^{-}$ & $(20: 0 / 18: 1)$ \\
\hline SM (d38:2) & & $+\mathrm{H}^{+}$ & $(18: 2 / 20: 0)$ \\
\hline SM (d40:0) & $+\mathrm{Na}^{+}$ & $+\mathrm{AcO}^{-},-\mathrm{CH}_{3}^{+}$ & $(18: 0 / 22: 0)$ \\
\hline SM (d40:1) & $-\mathrm{H}^{+},+\mathrm{H}^{+},+\mathrm{Na}^{+},+\mathrm{K}^{+}$ & $+\mathrm{H}^{+},+\mathrm{Na}^{+},+\mathrm{AcO}^{-},-\mathrm{CH}_{3}{ }^{+}$ & $(18: 1 / 22: 0) /(18: 0 / 22: 1)$ \\
\hline SM (d40:2) & & $+\mathrm{H}^{+}$ & $(18: 1 / 22: 1)$ \\
\hline SM (d41:2) & & $+\mathrm{H}^{+}$ & \\
\hline SM (d42:0) & & $+\mathrm{H}^{+},+\mathrm{Na}^{+},+\mathrm{AcO}^{-}$ & \\
\hline SM (d42:1) & $+\mathrm{H}^{+},+\mathrm{Na}^{+},+\mathrm{K}^{+}$ & $\begin{array}{l}+\mathrm{H}^{+},+\mathrm{Na}^{+},+\mathrm{K}^{+},+\mathrm{AcO}^{-} \\
-\mathrm{CH}_{3}^{+}\end{array}$ & $(18: 0 / 24: 0)$ \\
\hline SM (d42:2) & $+\mathrm{H}^{+},+\mathrm{Na}^{+},+\mathrm{K}^{+}$ & $+\mathrm{H}^{+},+\mathrm{Na}^{+},+\mathrm{AcO}^{-},-\mathrm{CH}_{3}{ }^{+}$ & $(18: 1 / 24: 1)$ \\
\hline
\end{tabular}

PE-Cer $(\mathrm{d} 38: 1)$

PE-Cer (d40:1)

PE-Cer (d42:1)

PE-Cer (d42:2)

PE-Cer (d44:1)

PE-Cer (d44:2)

PE-Cer (d44:3)

$-\mathrm{H}^{+}$
$-\mathrm{H}^{+}$
$-\mathrm{H}^{+}$
$-\mathrm{H}^{+}$
$-\mathrm{H}^{+}$
$-\mathrm{H}^{+}$
$-\mathrm{H}^{+}$
$-\mathrm{H}^{+}$

\begin{tabular}{lll}
\hline \hline & \\
LacCer (d32:0) & $+\mathrm{H}^{+}$ \\
LacCer (d42:1) & $+\mathrm{K}^{+}$ & \\
LacCer (d42:2) & $+\mathrm{K}^{+}$ & \\
\hline \hline
\end{tabular}

HexCer (d28:0)

HexCer (d30:0)

HexCer (d30:1)

HexCer (d34:0)

HexCer (d36:0)

HexCer (d36:1)

HexCer (d36:2)

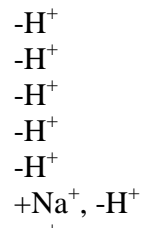

$-\mathrm{H}^{+}$

$\begin{array}{ll}-\mathrm{H}^{+} & (18: 0 / 18: 0) \\ +\mathrm{H}^{+},-\mathrm{H}^{+} & (18: 1 / 18: 0)\end{array}$


HexCer (d38:0)

HexCer (d38:1)

HexCer (d38:2)

HexCer (d40:0)

HexCer (d40:1)

HexCer (d40:2)

HexCer (d41:1)

HexCer (d42:0)

HexCer (d42:1)

HexCer (d42:2)

HexCer (d44:1)

HexCer (d44:2)
$+\mathrm{H}^{+},+\mathrm{Na}^{+},-\mathrm{H}^{-}$

$+\mathrm{H}^{+},+\mathrm{Na}^{+},-\mathrm{H}$

20:0/18:0) / (18:0/20:0)

$+\mathrm{Na}^{+},-\mathrm{H}^{+}$

$+\mathrm{Na}^{+},+\mathrm{K}^{+},-\mathrm{H}^{+}$

$+\mathrm{H}^{+}$

$(18: 1 / 20: 0)$

$(18: 1 / 20: 1)$

$+\mathrm{H}^{+},+\mathrm{Na}^{+},-\mathrm{H}^{-},+\mathrm{AcO}^{-}$

$+\mathrm{H}^{+},+\mathrm{Na}^{+},-\mathrm{H}^{-},+\mathrm{AcO}$

$+\mathrm{H}^{+}$

$-\mathrm{H}^{-},+\mathrm{AcO}^{-}$

$+\mathrm{H}^{+},+\mathrm{Na}^{+}, \mathrm{K}^{+},-\mathrm{H}^{-},+\mathrm{AcO}^{-}$

$+\mathrm{H}^{+},+\mathrm{Na}^{+},-\mathrm{H}^{-},+\mathrm{AcO}^{-}$

$+\mathrm{H}^{+},+\mathrm{Na}^{+},-\mathrm{H}^{-},+\mathrm{AcO}^{-}$

$+\mathrm{H}^{+},+\mathrm{Na}^{+},-\mathrm{H}^{-},+\mathrm{AcO}$

$-\mathrm{H}^{-},+\mathrm{AcO}^{-}$

(18:0/22:0)

$(18: 1 / 22: 1)$

$(18: 1 / 23: 0) /(23: 0 / 18: 1)$

$(18: 0 / 24: 0)$

(18:0/24:1) / (18:1/24:0)

$(18: 1 / 24: 1) /(18: 2 / 24: 0)$

$(18: 1 / 26: 0)$

$(18: 1 / 26: 1)$
SFT (d34:1)

SFT (d36:1)

SFT (d36:3)

SFT (d38:0)

SFT (d38:1)

SFT (d40:0)

SFT (d40:1)

SFT (d40:2)

SFT (d41:1)

SFT (d41:2)

SFT (d42:0)

SFT (d42:1)

SFT (d42:2)

SFT (d42:3)

SFT (d42:4)

SFT (d43:2)

SFT (d44:2)
$-\mathrm{H}^{+}$

$-\mathrm{H}^{+}$

$-\mathrm{H}^{+}$

$-\mathrm{H}^{+}$

$-\mathrm{H}^{+}$

$-\mathrm{H}^{+}$
$-\mathrm{H}^{+}$

$-\mathrm{H}^{+}$

$-\mathrm{H}^{+}$

$-\mathrm{H}^{+}$

$-\mathrm{H}^{+}$
$-\mathrm{H}^{+}$

$-\mathrm{H}^{+}$

$-\mathrm{H}^{+}$

$-\mathrm{H}^{+}$

$-\mathrm{H}^{+}$
$(18: 1 / 18: 0)$

(18:0/20:0)

$(18: 1 / 20: 0)$

$(18: 0 / 22: 0)$

(18:1/22:0)

$(18: 0 / 24: 0)$

$(18: 1 / 24: 0) /(18: 0 / 24: 1)$

$(18: 1 / 24: 1)$ 
Table S3. Triglyceride molecular species identified in the rat sciatic nerve by IMS or LCMS/MS

\begin{tabular}{|c|c|c|}
\hline \multirow[t]{2}{*}{ Species } & \multicolumn{2}{|c|}{ Adduct } \\
\hline & IMS & ESI \\
\hline TG $(38: 1)$ & & $+\mathrm{NH}_{4}^{+},+\mathrm{Na}^{+}$ \\
\hline TG $(40: 0)$ & & $+\mathrm{NH}_{4}^{+}$ \\
\hline TG $(40: 1)$ & & $+\mathrm{NH}_{4}^{+}$ \\
\hline TG (40:2) & & $+\mathrm{NH}_{4}^{+},+\mathrm{Na}^{+}$, \\
\hline TG (42:0) & & $+\mathrm{NH}_{4}^{+},+\mathrm{Na}^{+}$ \\
\hline TG (42:1) & & $+\mathrm{NH}_{4}^{+},+\mathrm{Na}^{+}$ \\
\hline TG (42:2) & & $+\mathrm{NH}_{4}^{+},+\mathrm{Na}^{+}$ \\
\hline TG (44:0) & & $+\mathrm{NH}_{4}^{+},+\mathrm{Na}^{+}$ \\
\hline TG (44:1) & & $+\mathrm{NH}_{4}^{+},+\mathrm{Na}^{+}$ \\
\hline TG (44:2) & & $+\mathrm{NH}_{4}^{+},+\mathrm{Na}^{+},+\mathrm{K}^{+}$ \\
\hline TG (44:3) & & $+\mathrm{NH}_{4}^{+},+\mathrm{Na}^{+}$ \\
\hline TG (44:4) & & $+\mathrm{NH}_{4}^{+},+\mathrm{Na}^{+}$, \\
\hline TG (44:5) & & $+\mathrm{NH}_{4}^{+}$ \\
\hline TG $(45: 1)$ & & $+\mathrm{NH}_{4}^{+}$ \\
\hline TG $(45: 2)$ & & $+\mathrm{NH}_{4}^{+}$ \\
\hline TG (46:0) & & $+\mathrm{NH}_{4}^{+}$ \\
\hline TG (46:1) & & $+\mathrm{NH}_{4}^{+},+\mathrm{Na}^{+}$ \\
\hline TG (46:2) & & $+\mathrm{NH}_{4}^{+},+\mathrm{Na}^{+},+\mathrm{K}^{+}$ \\
\hline TG (46:3) & & $+\mathrm{NH}_{4}^{+},+\mathrm{Na}^{+},+\mathrm{K}^{+}$ \\
\hline TG (46:4) & & $+\mathrm{NH}_{4}^{+},+\mathrm{Na}^{+}$ \\
\hline TG (46:5) & & $+\mathrm{NH}_{4}^{+}$ \\
\hline TG (46:6) & & $+\mathrm{NH}_{4}^{+}$ \\
\hline TG $(47: 0)$ & & $+\mathrm{NH}_{4}^{+}$ \\
\hline TG (47:1) & & $+\mathrm{NH}_{4}^{+}$ \\
\hline TG (47:2) & & $+\mathrm{NH}_{4}^{+}$ \\
\hline TG (47:3) & & $+\mathrm{NH}_{4}^{+}$ \\
\hline TG (48:0) & & $+\mathrm{NH}_{4}^{+},+\mathrm{Na}^{+}$ \\
\hline TG (48:1) & & $+\mathrm{NH}_{4}^{+},+\mathrm{Na}^{+}$ \\
\hline TG (48:2) & & $+\mathrm{NH}_{4}^{+},+\mathrm{Na}^{+}$ \\
\hline TG (48:3) & & $+\mathrm{NH}_{4}^{+},+\mathrm{Na}^{+},+\mathrm{K}^{+},+\mathrm{H}^{+}$ \\
\hline TG (48:4) & & $+\mathrm{NH}_{4}^{+},+\mathrm{Na}^{+},+\mathrm{K}^{+},+\mathrm{H}^{+}$ \\
\hline TG (48:5) & & $+\mathrm{NH}_{4}^{+},+\mathrm{Na}^{+}$ \\
\hline TG (48:6) & & $+\mathrm{NH}_{4}^{+}$ \\
\hline TG (49:0) & $+[\mathrm{MBT}+\mathrm{H}]^{+}$ & $+\mathrm{NH}_{4}^{+},+\mathrm{Na}^{+}$ \\
\hline TG (49:1) & $+[\mathrm{MBT}+\mathrm{H}]^{+}$ & $+\mathrm{NH}_{4}^{+},+\mathrm{Na}^{+}$ \\
\hline TG (49:2) & & $+\mathrm{NH}_{4}^{+},+\mathrm{Na}^{+}$ \\
\hline TG (49:3) & & $+\mathrm{NH}_{4}^{+},+\mathrm{Na}^{+}$ \\
\hline TG (49:4) & & $+\mathrm{NH}_{4}^{+}$ \\
\hline TG $(50: 0)$ & & $+\mathrm{NH}_{4}^{+},+\mathrm{Na}^{+}$ \\
\hline TG $(50: 1)$ & $+\mathrm{Na}^{+}$ & $+\mathrm{NH}_{4}^{+},+\mathrm{Na}^{+}$ \\
\hline TG $(50: 2)$ & $+[\mathrm{MBT}+\mathrm{H}]^{+},+\mathrm{Na}^{+}$ & $+\mathrm{NH}_{4}^{+},+\mathrm{Na}^{+}$ \\
\hline TG (50:3) & $+[\mathrm{MBT}+\mathrm{H}]^{+},+\mathrm{Na}^{+}$ & $+\mathrm{NH}_{4}^{+},+\mathrm{Na}^{+},+\mathrm{H}^{+}$ \\
\hline TG (50:4) & $+[\mathrm{MBT}+\mathrm{H}]^{+}$ & $+\mathrm{NH}_{4}^{+},+\mathrm{Na}^{+},+\mathrm{K}^{+},+\mathrm{H}^{+}$ \\
\hline TG (50:5) & $+[\mathrm{MBT}+\mathrm{H}]^{+}$ & $+\mathrm{NH}_{4}^{+},+\mathrm{Na}^{+},+\mathrm{K}^{+},+\mathrm{H}^{+}$ \\
\hline TG (50:6) & & $+\mathrm{NH}_{4}^{+}$ \\
\hline TG (50:7) & & $+\mathrm{NH}_{4}^{+}$ \\
\hline TG $(51: 0)$ & $+[\mathrm{MBT}+\mathrm{H}]^{+}$ & $+\mathrm{NH}_{4}^{+},+\mathrm{Na}^{+}$ \\
\hline TG $(51: 1)$ & $+[\mathrm{MBT}+\mathrm{H}]^{+}$ & $+\mathrm{NH}_{4}^{+},+\mathrm{Na}^{+}$ \\
\hline TG $(51: 2)$ & $+[\mathrm{MBT}+\mathrm{H}]^{+}$ & $+\mathrm{NH}_{4}^{+},+\mathrm{Na}^{+}$ \\
\hline TG $(51: 3)$ & & $+\mathrm{NH}_{4}^{+},+\mathrm{Na}^{+}$ \\
\hline TG $(51: 4)$ & & $+\mathrm{NH}_{4}^{+},+\mathrm{Na}^{+},+\mathrm{H}^{+}$ \\
\hline TG (51:5) & & $+\mathrm{NH}_{4}^{+},+\mathrm{Na}^{+}$ \\
\hline TG $(52: 0)$ & & $+\mathrm{NH}_{4}^{+},+\mathrm{Na}^{+}$ \\
\hline TG $(52: 1)$ & & $+\mathrm{NH}_{4}^{+},+\mathrm{Na}^{+}$ \\
\hline $\mathrm{TG}(52: 2)$ & $+[\mathrm{MBT}+\mathrm{H}]^{+},+\mathrm{Na}^{+}$ & $+\mathrm{NH}_{4}^{+},+\mathrm{Na}^{+}$ \\
\hline TG $(52: 3)$ & $+[\mathrm{MBT}+\mathrm{H}]^{+},+\mathrm{Na}^{+}$ & $+\mathrm{NH}_{4}^{+},+\mathrm{Na}^{+}$ \\
\hline TG (52:4) & $+[\mathrm{MBT}+\mathrm{H}]^{+},+\mathrm{Na}^{+}$ & $+\mathrm{NH}_{4}^{+},+\mathrm{Na}^{+},+\mathrm{H}^{+}$ \\
\hline TG $(52: 5)$ & $+[\mathrm{MBT}+\mathrm{H}]^{+},+\mathrm{Na}^{+}$ & $+\mathrm{NH}_{4}^{+},+\mathrm{Na}^{+},+\mathrm{K}^{+},+\mathrm{H}^{+}$ \\
\hline TG (52:6) & $+[\mathrm{MBT}+\mathrm{H}]^{+}$ & $+\mathrm{NH}_{4}^{+},+\mathrm{Na}^{+},+\mathrm{K}^{+},+\mathrm{H}^{+}$ \\
\hline
\end{tabular}




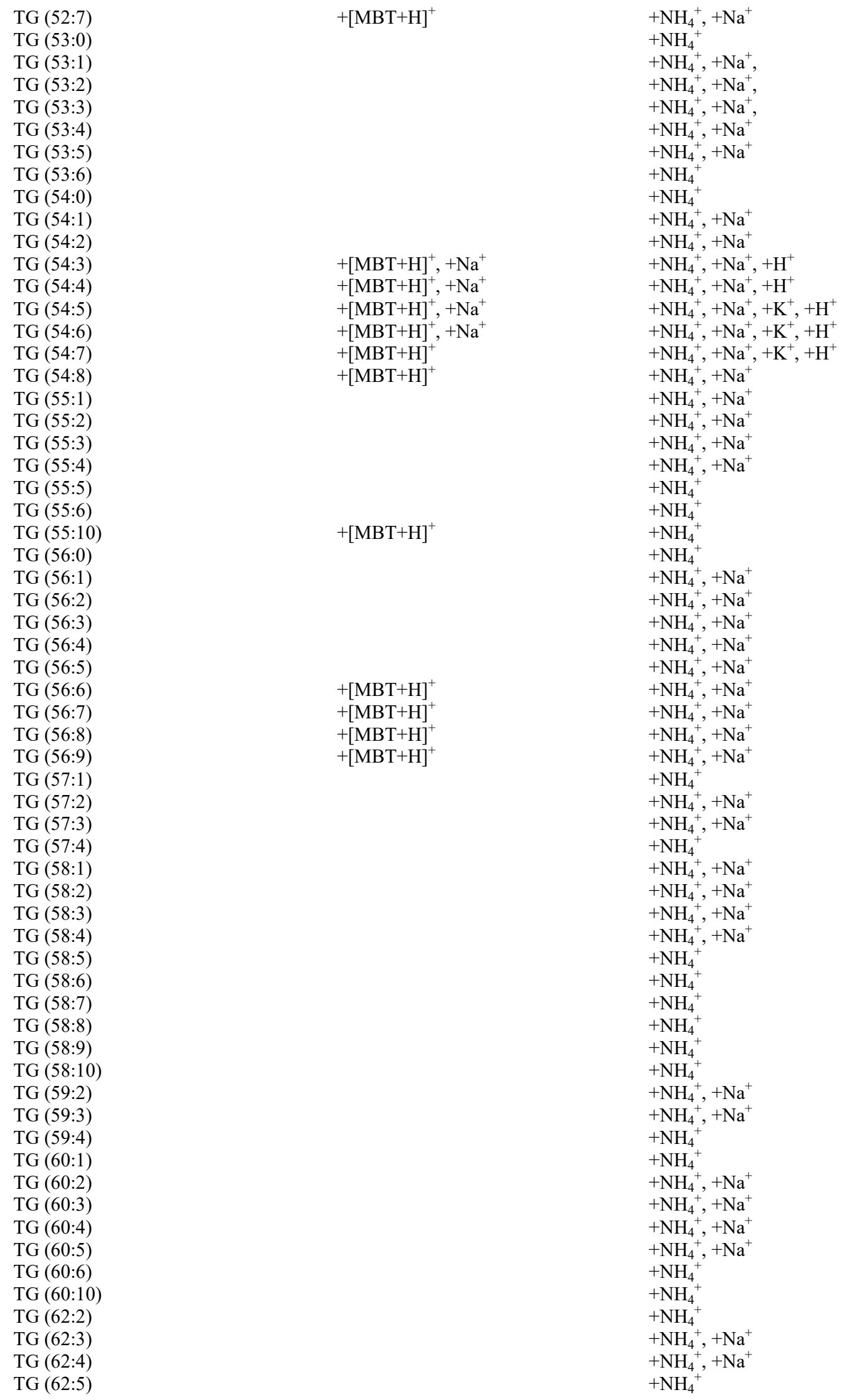


Table S4. Diglyceride molecular species identified in the rat sciatic nerve by IMS or LCMS/MS.

\begin{tabular}{|c|c|c|c|}
\hline \multirow[t]{2}{*}{ Species } & \multicolumn{2}{|c|}{ Adduct } & \multirow[t]{2}{*}{ Tentative Structure } \\
\hline & IMS & ESI & \\
\hline $\begin{array}{l}\text { DG }(32: 1) \\
\text { DG }(34: 1) \\
\text { DG }(34: 2) \\
\text { DG }(36: 2) \\
\text { DG }(36: 3) \\
\text { DG }(36: 4) \\
\text { DG }(38: 4)\end{array}$ & $+\mathrm{Na}^{+}$ & $\begin{array}{l}+\mathrm{Na}^{+} \\
+\mathrm{Na}^{+} \\
+\mathrm{Na}^{+},+\mathrm{K}^{+} \\
+\mathrm{Na}^{+} \\
+\mathrm{Na}^{+},+\mathrm{K}^{+},+\mathrm{H}^{+},+\mathrm{NH}_{4}^{+} \\
+\mathrm{Na}^{+},+\mathrm{H}^{+} \\
+\mathrm{Na}^{+},+\mathrm{H}^{+},+\mathrm{NH}_{4}^{+}\end{array}$ & $\begin{array}{l}(16: 0 / 18: 1) \\
(16: 0 / 18: 2) \\
(18: 0 / 18: 2) \\
(18: 1 / 18: 2) \\
(18: 2 / 18: 2) \\
(18: 0 / 20: 4)\end{array}$ \\
\hline $\begin{array}{l}\text { DG }(\mathrm{O}-30: 0) \\
\text { DG }(\mathrm{O}-32: 0) \\
\text { DG }(\mathrm{O}-32: 1) \\
\text { DG }(\mathrm{O}-32: 2) \\
\text { DG }(\mathrm{O}-34: 0) \\
\text { DG }(\mathrm{O}-34: 1) \\
\text { DG }(\mathrm{O}-34: 2) \\
\text { DG }(\mathrm{O}-34: 3) \\
\text { DG }(\mathrm{O}-34: 4)) \\
\text { DG }(\mathrm{O}-36: 4)\end{array}$ & $\begin{array}{l}+\mathrm{Na}^{+} \\
+\mathrm{Na}^{+} \\
+\mathrm{Na}^{+} \\
+\mathrm{Na}^{+} \\
+\mathrm{Na}^{+} \\
+\mathrm{Na}^{+} \\
+\mathrm{Na}^{+} \\
+\mathrm{Na}^{+} \\
+\mathrm{Na}^{+} \\
+[\mathrm{MBT}+\mathrm{H}]^{+}\end{array}$ & & \\
\hline
\end{tabular}


Table S5. Comparison between experimental and theoretical $\mathrm{m} / \mathrm{z}$ values for all the species detected in MALDI-IMS, in both positive and negative ion modes.

\begin{tabular}{|c|c|c|c|c|}
\hline $\mathbf{m} / \mathbf{z} \exp$ & Lipid & $\mathbf{m} / \mathbf{z}$ teor & error (Da) & error (PPM) \\
\hline 496.3411 & [Lyso-PC_16:0 + H]+ & 496.3404 & -0.00070 & -1.41 \\
\hline 522.3561 & [Lyso-PC_18:1 + H]+ & 522.3560 & -0.00008 & -0.15 \\
\hline 549.4883 & [DG_O-30:0 + Na]+ & 549.4858 & -0.00249 & -4.53 \\
\hline 573.4879 & {$[$ DG_O-32:2 + Na]+/ [DG_P-32:1 + Na]+ } & 573.4858 & -0.00207 & -3.60 \\
\hline 575.5050 & {$[$ DG_O-32:1 $+\mathrm{Na}]+/[$ DG_P-32:0 $+\mathrm{Na}]+$} & 575.5015 & -0.00347 & -6.03 \\
\hline 577.5195 & {$[$ DG_O-32:0 + Na]+ } & 577.5171 & -0.00233 & -4.03 \\
\hline 581.3148 & [Lyso-PI_O-18:2 - H]- & 581.3092 & -0.00566 & -9.74 \\
\hline 597.2782 & [PG_24:6 - H]- & 597.2829 & 0.00474 & 7.93 \\
\hline 597.4883 & [DG_O-34:4 + Na]+/ [DG_P-34:3 + Na]+ & 597.4858 & -0.00243 & -4.06 \\
\hline 599.3253 & [Lyso-PI_18:0 - H]- & 599.3197 & -0.00554 & -9.24 \\
\hline 599.5040 & [DG_O-34:3 + Na]+/ [DG_P-34:2 + Na]+ & 599.5015 & -0.00251 & -4.18 \\
\hline 601.5206 & {$[$ DG_O-34:2 + Na]+/ [DG_P-34:1 + Na] +} & 601.5171 & -0.00351 & -5.83 \\
\hline 603.5355 & {$[$ DG_O-34:1 + Na]+/ [DG_P-34:0 + Na] +} & 603.5328 & -0.00276 & -4.57 \\
\hline 605.5509 & {$\left[\mathrm{DG} \_\mathrm{O}-34: 0+\mathrm{Na}\right]+$} & 605.5484 & -0.00242 & -4.00 \\
\hline 610.2753 & [PS_24:6 - H]- & 610.2782 & 0.00287 & 4.70 \\
\hline 612.2928 & [PS_24:5 - H]- & 612.2939 & 0.00103 & 1.68 \\
\hline 614.3035 & [PS_24:4 - H]- & 614.3095 & 0.00604 & 9.84 \\
\hline 615.4979 & $*\left[D G \_34: 2+\mathrm{Na}\right]+$ & 615.4964 & -0.00149 & -2.43 \\
\hline 616.4748 & $+[$ CerP_d34:1 - H] - & 616.4706 & -0.00425 & -6.90 \\
\hline 616.4748 & $+[$ HexCer_d28:0 - H] - & 616.4788 & 0.00400 & 6.48 \\
\hline 642.4909 & $+[$ CerP_d36:2 - H] - & 642.4862 & -0.00466 & -7.25 \\
\hline 642.4909 & $+[$ HexCer_d30:1 - H] - & 642.4945 & 0.00364 & 5.67 \\
\hline 644.5057 & $+[$ CerP_d36:1 - H]- & 644.5019 & -0.00381 & -5.91 \\
\hline 644.5057 & $+[$ HexCer_d30:0 - H] - & 644.5101 & 0.00444 & 6.89 \\
\hline 647.4688 & [PA_32:0 - H]- & 647.4652 & -0.00363 & -5.61 \\
\hline 671.4696 & [PA_34:2 - H]- & 671.4652 & -0.00439 & -6.53 \\
\hline 673.4856 & [PA_34:1 - H]- & 673.4809 & -0.00473 & -7.03 \\
\hline 687.5476 & [PE-Cer_d36:1 - H]- & 687.5441 & -0.00347 & -5.05 \\
\hline 697.4783 & [PA_34:1 + Na]+ & 697.4785 & 0.00012 & 0.17 \\
\hline 699.5009 & [PA_36:2 - H]- & 699.4965 & -0.00441 & -6.30 \\
\hline 700.5684 & $+[$ CerP_d40:1 - H] - & 700.5645 & -0.00395 & -5.64 \\
\hline 700.5684 & $+[$ HexCer_d34:0 - H] - & 700.5727 & 0.00430 & 6.14 \\
\hline 701.5163 & [PA_36:1 - H]- & 701.5122 & -0.00413 & -5.88 \\
\hline 703.5756 & $*[$ SM_d $34: 1+\mathrm{H}]+$ & 703.5754 & -0.00022 & -0.31 \\
\hline 706.5383 & {$[$ PC_30:0 + H]+ } & 706.5388 & 0.00041 & 0.58 \\
\hline 713.4537 & [PA_34:1+K]+ & 713.4524 & -0.00133 & -1.87 \\
\hline 715.5786 & [PE-Cer_d38:1 - H]- & 715.5754 & -0.00317 & -4.43 \\
\hline 716.5273 & [PE_34:1 - H]- & 716.5231 & -0.00427 & -5.96 \\
\hline 718.5432 & [PE_34:0 - H]- & 718.5387 & -0.00446 & -6.21 \\
\hline 723.4945 & $*[$ PA_36:2 + Na]+ & 723.4941 & -0.00036 & -0.50 \\
\hline 723.5006 & [PA_38:4 - H]- & 723.4965 & -0.00406 & -5.61 \\
\hline 724.5672 & [CerP_d42:3 - H]- & 724.5645 & -0.00269 & -3.71 \\
\hline
\end{tabular}




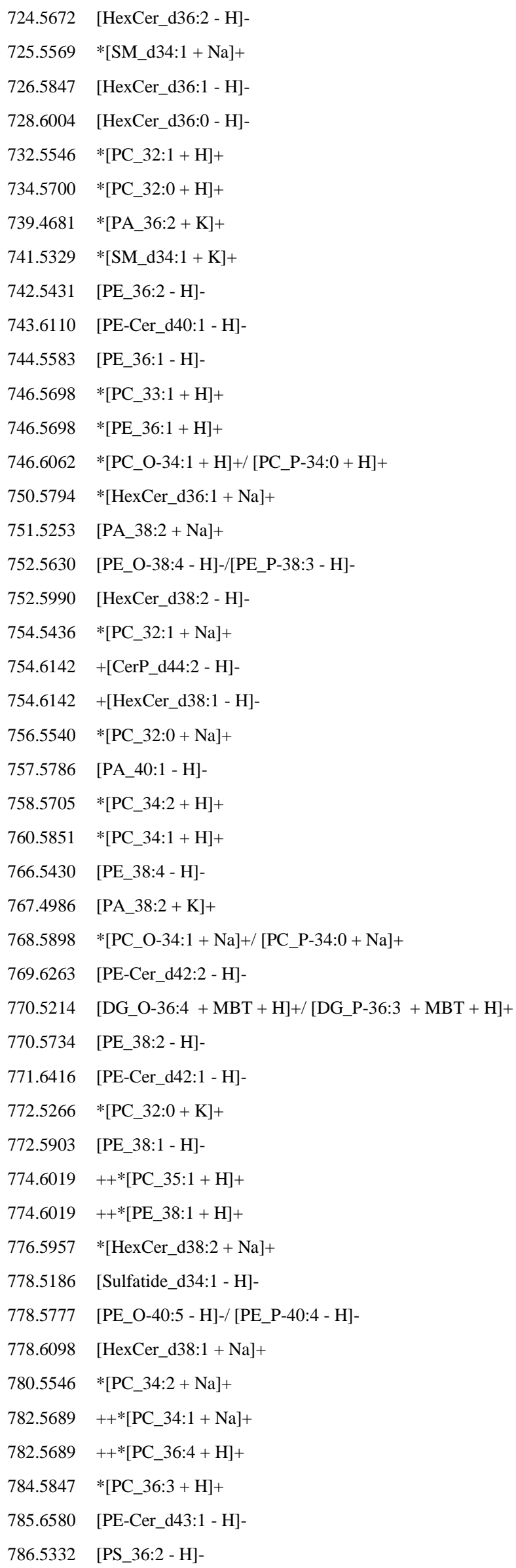




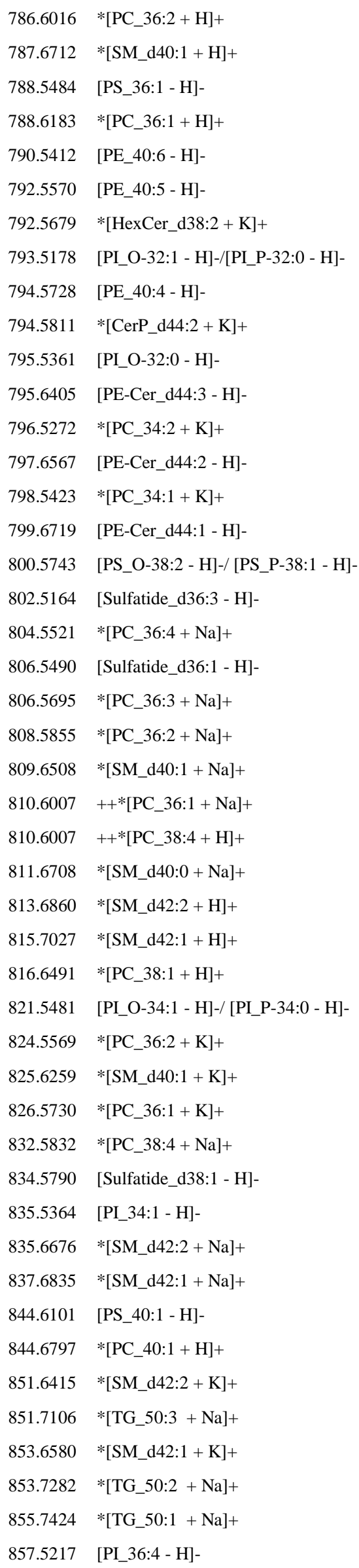

\begin{tabular}{|c|c|c|}
\hline 36.6014 & -0.00020 & -0.26 \\
\hline 787.6693 & -0.00189 & -2.40 \\
\hline 788.5443 & -0.00413 & -5.24 \\
\hline 788.6170 & -0.00130 & -1.65 \\
\hline 790.5387 & -0.00245 & -3.10 \\
\hline 2.5544 & -0.00263 & -3.31 \\
\hline 792.5755 & 0.00766 & 9.67 \\
\hline 793.5232 & 0.00538 & 6.77 \\
\hline 794.5700 & -0.00279 & -3.51 \\
\hline 794.5829 & 0.00186 & 2.34 \\
\hline 95.5388 & 0.00273 & 3.43 \\
\hline 795.6380 & -0.00247 & -3.10 \\
\hline 796.5259 & -0.00126 & -1.58 \\
\hline 797.6536 & -0.00307 & -3.85 \\
\hline 798.5416 & -0.00069 & -0.87 \\
\hline 799.6693 & -0.00265 & -3.31 \\
\hline 800.5806 & 0.00631 & 7.89 \\
\hline 802.5139 & -0.00251 & -3.13 \\
\hline 804.5520 & -0.00012 & -0.15 \\
\hline 806.5452 & -0.00376 & -4.66 \\
\hline 806.5677 & -0.00180 & -2.23 \\
\hline 808.5833 & -0.00222 & -2.75 \\
\hline 809.6512 & 0.00040 & 0.49 \\
\hline 810.5990 & -0.00174 & -2.15 \\
\hline 810.6014 & 0.00067 & 0.82 \\
\hline 811.6669 & -0.00392 & -4.83 \\
\hline 813.6849 & -0.00106 & -1.30 \\
\hline 815.7006 & -0.00208 & -2.55 \\
\hline 816.6483 & -0.00084 & -1.02 \\
\hline 821.5545 & 0.00644 & 7.84 \\
\hline 824.5572 & 0.00037 & 0.45 \\
\hline 825.6252 & -0.00072 & -0.87 \\
\hline 826.5729 & -0.00011 & -0.14 \\
\hline 832.5833 & 0.00007 & 0.08 \\
\hline 834.5765 & -0.00251 & -3.01 \\
\hline 835.5338 & -0.00260 & -3.11 \\
\hline 835.6669 & -0.00070 & -0.84 \\
\hline 837.6825 & -0.00098 & -1.17 \\
\hline 844.6069 & -0.00323 & -3.82 \\
\hline 844.6796 & -0.00013 & -0.16 \\
\hline 851.6408 & -0.00071 & -0.83 \\
\hline 851.7104 & -0.00018 & -0.21 \\
\hline 353.6565 & -0.00153 & -1.80 \\
\hline 853.7261 & -0.00209 & -2.45 \\
\hline 855.7417 & -0.00070 & -0.82 \\
\hline 7.5181 & -0.00356 & -4.15 \\
\hline
\end{tabular}




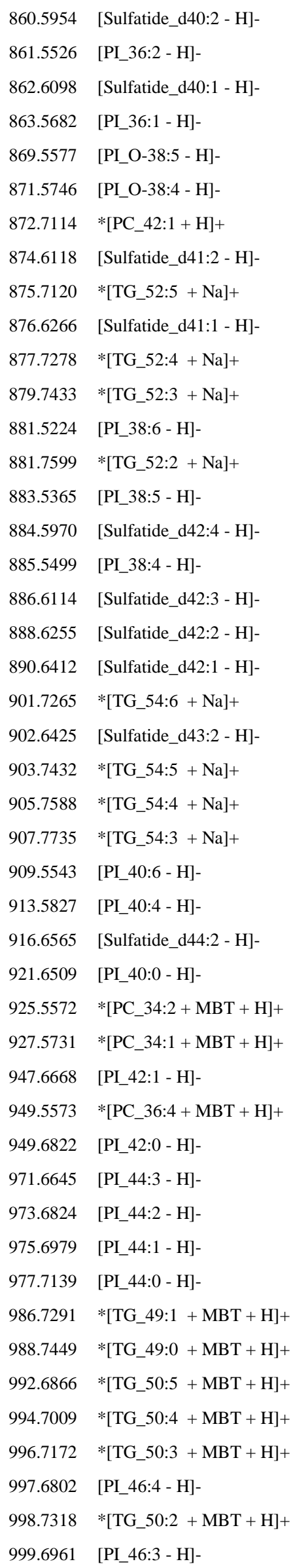

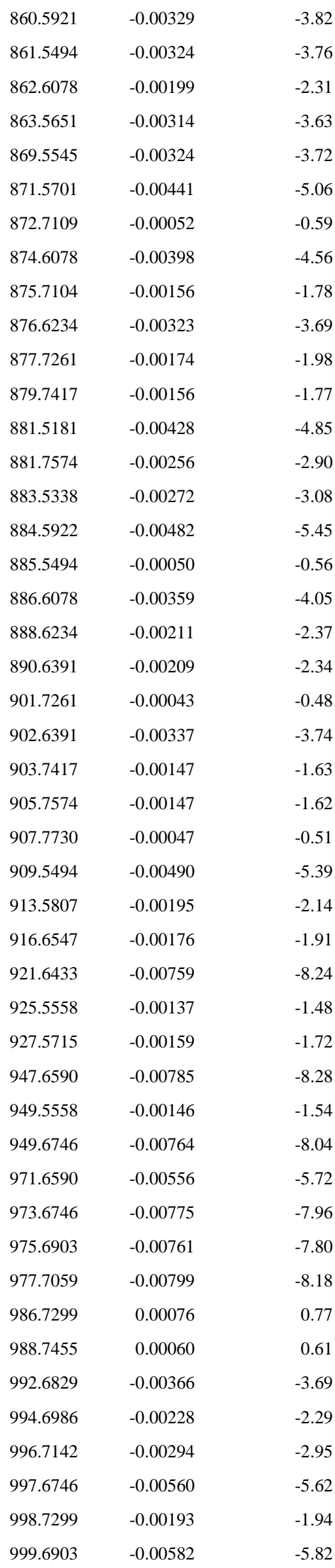




\begin{tabular}{|c|c|c|c|c|}
\hline 1001.7121 & [PI_46:2 - H]- & 1001.7059 & -0.00617 & -6.15 \\
\hline 1010.6949 & {$[\operatorname{LacCer}(\mathrm{d} 42: 2)+\mathrm{K}]+$} & 1010.6904 & -0.00451 & 0.00 \\
\hline 1012.7105 & {$[\operatorname{LacCer}(\mathrm{d} 42: 1)+\mathrm{K}]+$} & 1012.7061 & -0.00438 & 0.00 \\
\hline 1012.7463 & $*[$ TG_51:2 + MBT $+\mathrm{H}]+$ & 1012.7455 & -0.00078 & -0.77 \\
\hline 1014.7604 & $*[$ TG_51:1 + MBT $+\mathrm{H}]+$ & 1014.7612 & 0.00078 & 0.77 \\
\hline 1016.6854 & $*\left[\mathrm{TG} \_52: 7+\mathrm{MBT}+\mathrm{H}\right]+$ & 1016.6829 & -0.00248 & -2.44 \\
\hline 1016.7774 & $*[$ TG_51:0 + MBT $+\mathrm{H}]+$ & 1016.7768 & -0.00054 & -0.54 \\
\hline 1018.7004 & $*[$ TG_52:6 + MBT + H] + & 1018.6986 & -0.00181 & -1.78 \\
\hline 1020.7167 & $*[$ TG_52:5 + MBT $+\mathrm{H}]+$ & 1020.7142 & -0.00247 & -2.42 \\
\hline 1022.7309 & $*[$ TG_52:4 + MBT $+\mathrm{H}]+$ & 1022.7299 & -0.00102 & -0.99 \\
\hline 1024.7470 & $*[$ TG_52:3 + MBT $+\mathrm{H}]+$ & 1024.7455 & -0.00142 & -1.39 \\
\hline 1026.7629 & $*[$ TG_52:2 + MBT $+\mathrm{H}]+$ & 1026.7612 & -0.00168 & -1.64 \\
\hline 1042.7007 & $*[$ TG_54:8 + MBT $+\mathrm{H}]+$ & 1042.6986 & -0.00206 & -1.98 \\
\hline 1044.7165 & $*[$ TG_54:7 + MBT $+\mathrm{H}]+$ & 1044.7142 & -0.00227 & -2.17 \\
\hline 1046.7312 & $*[$ TG_54:6 + MBT $+\mathrm{H}]+$ & 1046.7299 & -0.00127 & -1.21 \\
\hline 1048.7473 & $*[$ TG_54:5 + MBT $+\mathrm{H}]+$ & 1048.7455 & -0.00173 & -1.64 \\
\hline 1050.7628 & $*\left[\mathrm{TG} \_54: 4+\mathrm{MBT}+\mathrm{H}\right]+$ & 1050.7612 & -0.00165 & -1.57 \\
\hline 1052.6892 & $*[$ TG_55:10 + MBT + H] + & 1052.6829 & -0.00627 & -5.96 \\
\hline 1052.7784 & $*[$ TG_54:3 + MBT $+\mathrm{H}]+$ & 1052.7768 & -0.00153 & -1.45 \\
\hline 1068.7158 & $*[$ TG_56:9 + MBT $+\mathrm{H}]+$ & 1068.7142 & -0.00158 & -1.48 \\
\hline 1070.7332 & $*[$ TG_56:8 + MBT $+\mathrm{H}]+$ & 1070.7299 & -0.00330 & -3.08 \\
\hline 1072.7496 & $*[$ TG_56:7 + MBT $+\mathrm{H}]+$ & 1072.7455 & -0.00408 & -3.81 \\
\hline 1074.7631 & $*\left[\mathrm{TG} \_56: 6+\mathrm{MBT}+\mathrm{H}\right]+$ & 1074.7612 & -0.00186 & -1.73 \\
\hline
\end{tabular}

Table S6. Relative abundance of species containing odd chains in each tissue of rat sciatic nerve.

\begin{tabular}{lcccc}
\hline \hline & Adipose tissue & Conjunctive tissue & Fibres & Tissue Average \\
\hline$\%$ Odd chains & 2.4 & 1.8 & 1.5 & 1.8 \\
\hline \hline
\end{tabular}

